NBER WORKING PAPER SERIES

\author{
TRANSACTION COSTS IN DEALER \\ MARKETS: EVIDENCE FROM \\ THE LONDON STOCK EXCHANGE
}

Peter C. Reiss

Ingrid $\mathbf{M}$. Werner

Working Paper No. 4727

\author{
NATIONAL BUREAU OF ECONOMIC RESEARCH \\ 1050 Massachusetts Avenue \\ Cambridge, MA 02138 \\ May 1994
}

We thank Stephen Wells and Graham Hart of the London Stock Exchange's Quality of Markets Group for providing data and answering questions. Goldman Sachs, Preferred Technology, Salomon Brothers, and UBS graciousiy granted us access to their trading operations. We had the good fortune to observe Xavier Rolet, Richard O'Hare and Dan Pizza make markets and to discuss the UK securities industry with George Gray. Kathieen Tyson of the UK Securities Investment Board explained the intricacies of UK security regulations. Helpful comments were provided by participants at the NBER conterence on "Industrial Organization and Regulation of Securities Markets," Key Largo, January 1994, and the conference on "Competition for Orderflow," University of Memphis, March 1994. Special thanks go to our discussants Bemard Black and Douglas Foster. The Stanford Graduate School of Business funded part of this research through its Financial Services Research Initiative and a Robert M. and Ann T. Bass Faculty Fellowship to Werner. Werner also acknowiedges her stay at the London School of Economics. The authors are responsible for the paper's analysis and conclusions. This paper is part of NBER's research programs in Asset Pricing and Industrial Organization. Any opinions expressed are those of the authors and not those of the National Bureau of Economic Research. 


\section{TRANSACTION COSTS IN DEALER \\ MARKETS: EVIDENCE FROM THE LONDON STOCK EXCHANGE}

\section{ABSTRACT}

This paper describes regularities in the intraday spreads and prices quoted by dealers on the London Stock Exchange. It develops a measure of spread-related transaction costs, one that recognizes dealers' willingness to price trades within their quoted spreads. This measure of transaction costs shows that trading costs are systematically related to a trade's size, characteristics of the trading counterparties, and security characteristics. Customers pay the full spread on small trades while medium to large trades receive more favorable execution. Market makers only discount very large customer trades while dealers regularly discount medium to large trades. Inter-dealer trades generally receive favorable execution, and discounts increase in size. Market makers do not discount trades with each other over the phone, but do discount when trading anonymously using inter-dealer-brokers. Quoted and touch spreads are falling in the number of market makers. The rate of decline is interpreted as reflecting economies of scale in market making.

Peter C. Reiss Graduate School of Business Stanford University

Stanford, CA 94305-5015 and NBER

\author{
Ingrid M. Werner \\ Graduate School of Business \\ Stanford University \\ Stanford, CA 94305-5015
}




\section{Introduction}

New electronic trading technologies have drastically reduced the costs of financial transactions and put tremendous pressure on financial exchanges to lower their costs. The London Stock Exchange [LSE] is a prominent example of the changes being made in response to this pressure. In 1986, the LSE switched from a closed, floor-based, brokerdealer market to an open electronic quotation system dubbed SEAQ. The LSE's SEAQ system operates much like the NASD's NASDAQ dealer system. On SEAQ, competing market makers post bid and ask prices and guaranteed trading volumes. Although SEAQ also displays trade information, brokers and dealers still negotiate trades by phone. Besides changing its systems, the LSE enacted new rules designed to encourage competition and narrow quoted spreads. These included the elimination of fixed commissions and member entry barriers, and the adoption of best execution rules. The Exchange also imposed what it calls mandatory quote volumes. Mandatory quote volumes require market makers to accept trades equalling two or more percent of a security's average daily trading volume. To reduce the capital risks associated with large trades, the Exchange granted market makers the right to delay their disclosure. During 1991, SEAQ market makers could delay releasing information on large trades for up to ninety minutes. Currently, they may delay disclosing them for up to one week!

The LSE's emphasis on liquidity over transparency has renewed debate about whether such rules affect the costs of financial transactions. A recent IOSCO report (1993) observed that few studies have studied the determinants of transaction costs in dealership markets. Several empirical studies report substantial inter-security and inter-day variation in NASDAQ and SEAQ dealer spreads. Few examine the relationship between spreads and transaction prices, or consider how exchange rules might affect spreads. This paper uses newly available SEAQ intra-day quotation and transaction data to analyze the relationship between investor transaction costs and best bid-ask spreads. Using unique information in the SEAQ data, we show that LSE rules lead dealers to offer systematic discounts from posted prices. These discounts vary across traders, securities and trade characteristics.

We also argue that conventional transaction cost measures do not recognize important institutional features of dealership markets. On SEAQ, these include mandatory quote volumes and best execution rules. These rules affect dealers' quoted prices and their willingness to offer traders discounts. Contrary to the assumptions of many theoretical 
models, SEAQ market makers do not compete by narrowing (symmetric) quotes. Indeed, they almost never narrow the quoted spread between their bid and ask prices. They instead compete by positioning their bid or ask on or at the market bid or offer. Curiously, they may maintain these positions for hours or days, offering traders discounts instead of changing what they advertise on SEAQ screens. We find, as some theoretical models do, that dealer discounts usually increase with the size of a trade. We also find puzzles. Surprisingly, discounts for customers, brokers and market makers all decrease with market maker concentration and increase with market depth. These heterogeneities raise new theoretical and empirical questions about how dealer competition affects the relation between quoted prices and transaction prices.

Section 2 begins with a review of prior research on transaction costs, particularly transaction costs in dealer markets. We then show that several standard transaction cost measures may over- or under-represent dealer discounts from quoted spreads. We illustrate our arguments using SEAQ and NASDAQ intra-day trade and quotation data for Cadbury \& Schweppes, a heavily traded FTSE-100 equity. We find that SEAQ Cadbury quoted bid-ask spreads are slightly higher than NASDAQ spreads. More notable are differences in the price discounts offered by NASDAQ and SEAQ market makers. Though some research suggests that dealers only offer other dealers discounts from quoted prices, this is not true on SEAQ. SEAQ market makers grant discounts to medium and large retail orders more often than they do to each other. The median retail discount increases uniformly in order size, and applies to many orders larger than the Cadbury mandatory quote volume. We conclude the Cadbury example by developing a new measure of transaction costs, what we term the adjusted apparent spread. This measure reveals how dealers regularly vary spreads and discounts with the characteristics of trades. We estimate these adjusted apparent spreads using quantile regression techniques. These regressions flexibly describe the distribution of Cadbury discounts conditional on trade size and the identities of traders.

Section 3 analyzes 1991 quotation data for 1,887 UK and Irish equities to isolate interand intra-security variations in quoted prices. We find striking variation. Best bid-ask spreads range from less than one percent of share value to over fifty percent! Much of this variation is systematic. As on NASDAQ, quoted and best bid-ask spreads decline as the number of posting market makers increases. It also appears that the greater the capital risk associated with mandatory quote volumes, the more market makers widen their spreads. Dealer participation in actively "making the market" also falls as turnover 
increases. At any instant, nearly one-third of the market makers in a heavily traded equity post non-competitive prices. While our empirical methods do not disentangle the interplay between dealer concentration, spreads and volume, we examine whether trade size economies or order processing costs could explain these correlations. We find limited evidence that they do.

Section 4 analyzes a smaller sample of sixty SEAQ securities divided equally among three market capitalization classes. Conventional transaction cost statistics imply that each capitalization class has best bid-ask spreads comparable to NASDAQ spreads. Our conditional apparent spread measures show that transaction costs for individual securities differ substantially because of differences in the extent of discounting. Some of these differences occur because trade characteristics differ across market capitalization classes. For instance, FTSE-100 equities appear to have lower transaction costs because they have relatively more discounted inter-dealer trades. Other differences occur because market makers and brokers charge different customers different prices. The median customer trade is never discounted by market makers, but non-registered dealers give their median customers substantial discounts. We also show that market makers appear unwilling to give each other price breaks over the phone, but do when trading anonymously through inter-dealer brokers. Finally, we find some evidence that large orders receive greater discounts in less concentrated markets.

\section{Measuring Transaction Costs in Dealer Markets}

Investors incur several types of transaction costs each time they trade. These include commissions, differences between purchase and sale prices, and costs related to the price impact of trades. This paper exclusively analyzes differences between purchase and sales prices. We analyze spreads largely because the LSE does not regularly monitor commissions. In 1991, the Exchange estimated that commissions on trades between $£ 50,001-£ 100,000$ averaged 0.23 percent of price. Smaller round lots paid as much as 6.11 percent, and orders greater than $£ 1,000,000$ an average of 0.15 percent. ${ }^{1}$ These commissions only accentuate the spread-related size discounts we report below.

Prior theoretical and empirical market microstructure research has devoted considerable effort to modelling how market makers set spreads. Most conceptual models focus on a single market maker or specialist. These models show how factors such as limit order competition and a trade's size affect specialists' spreads. Many models, for in- 
stance, conclude that larger trades will be charged larger spreads. In inventory models this occurs because of inventory risk; in adverse selection models it occurs because large trades move prices. ${ }^{2}$

Empirical research on the determinants of spreads has struggled with the question of how to estimate transaction costs when transaction prices differ from dealers' posted quotes. Much of this research relies on NYSE intra-day transaction and quotation data. This research reveals that specialists vary their spreads in systematic ways (e.g., Brock and Kleidon (1992), McInish and Wood (1992), and Foster and Viswanathan (1993).) These findings have inspired new theoretical models of trade between different types of investors and market intermediaries.

Fewer papers have modelled the behavior of market makers in dealer quotation markets. Models by Ho and Stoll (1983), Grossman and Miller (1988), Glosten (1992), Madhavan (1992), Biajs (1993), Dennert (1993), and others show that strategic interactions among market makers can considerably complicate relationships between spreads and trade characteristics. Consider, for example, the conclusion cited above that specialists will charge large orders larger spreads. In dealership markets, such a policy would give large traders an incentive to split trades among dealers. Absent centralized information on the identities of traders, dealers will have a harder time identifying and pricing informed trades. Recently, Glosten (1993) has shown that minimum tick sizes may similarly constrain dealers' abilities to charge large orders high spreads. His model also provides some intuition for how SEAQ mandatory quote volumes may affect spreads. By forcing dealers to accept large and small trades at the same price, SEAQ mandatory quote volume rules give market makers incentives to widen spreads. By widening spreads, SEAQ market makers can protect themselves against inventory imbalances and informed trades while simultaneously retaining an option to offer execution within their guaranteed quotes. What is unclear is whether competition will force market makers to offer discounts. Studies of NYSE specialists suggest that they only offer small orders discounts. Large floor trades receive less favorable execution at or outside the bid and ask. $^{3}$

Few studies have examined the relation between quoted prices and transaction prices in dealer markets. Some descriptions of NASDAQ claim that only inter-dealer or Instinet transactions trade within the quoted spread. A recent study by the LSE's Quality of Markets Group estimated that nearly thirty-five percent of SEAQ trades occur within 
the best-bid ask spread. ${ }^{4}$ Some researchers speculate that these are inter-dealer trades. Others interpret this statistic as evidence that SEAQ quoted prices do not contain much information. The LSE's Quality of Markets Review and Neuberger and Schwartz (1989) report that not all trades within the spread are dealer trades. The analysis below shows that while many trades within the best bid-ask spread on SEAQ are inter-dealer trades, large customer trades also receive favorable execution. Small and very large trades usually do not. ${ }^{5}$ These comparisons do not show, however, whether other characteristics also affect discounts. We now explore whether trader identities and other factors affect SEAQ transaction costs.

\section{Cadbury 8 Schweppes - An Introductory Example}

We begin our analysis of SEAQ transaction costs by analyzing what conventional transaction cost measures reveal about the cost of trading Cadbury \& Schweppes, a heavily traded FTSE-100 stock. We analyze Cadbury for several reasons. First, by focusing on a single security we can more clearly describe SEAQ trading rules that might affect transaction costs. Second, during the period we study Cadbury \& Schweppes market makers had to accept trades as large as $£ 100,000$ - quite large by NASDAQ and SEAQ standards. Third, Cadbury \& Schweppes shares also trade on NASDAQ as American Depository Receipts. This dual trading of Cadbury shares allows us to compare transaction costs in two very similar dealer markets.

The Cadbury SEAQ data come from separate settlement and quotation records maintained by the LSE. The NASDAQ data come from the Institute for the Study of Securities Markets. Subsequent sections and a Data Appendix describe these data in greater detail. Table 1 provides conventional descriptive statistics on Cadbury transactions during 1991. The top section of the table contains transaction cost statistics developed in prior studies. Following convention, we express each as a percentage of Cadbury's average share price. For comparison, we also convert each to a pound sterling estimate of the spread cost on a median size SEAQ trade. (Roughly 1,500 shares.) These measures convey very different impressions of transaction costs, both on the same exchange (reading down columns) or between exchanges (reading across pairs of columns). SEAQ spread-related cost estimates range from 0.54 to 0.85 percent, a difference of $£ 18$ on the average trade. NASDAQ cost estimates range from 0.47 to 0.71 , a difference of $£ 13.5$. On both exchanges, the difference between the best bid and ask is clearly a weak upper bound on costs. The average discount from the best bid or ask on SEAQ is 0.07 percent, or roughly 
$\mathcal{E} 4.4$ on a median-size trade.

Comparing spreads across exchanges, we find that NASDAQ has lower spread-related transaction costs. The best bid-ask statistics show that on a median-size SEAQ trade, NASDAQ traders save $\mathfrak{E} 8.2$ ! The value-weighted NASDAQ measures also show that large NASDAQ trades receive substantial discounts from quoted prices. When one excludes trades outside the best bid-ask and weights spreads by value, however, it appears that there is not a substantial difference between SEAQ and NASDAQ. The distribution of trade values about the best bid-ask prices provides one possible explanation for the difference narrowing. Although SEAQ has wider best bid-ask spreads, a larger percentage of large SEAQ trades go through inside the best bid-ask prices. The difference between the SEAQ value-weighted and the unweighted effective spreads suggests that SEAQ transaction costs fall substantially with the size of a trade. The difference between the effective spreads of $£ 5.3$ roughly equals the commission on a $£ 2,300$ trade.

Although the statistics in Table 1 suggest that Cadbury traders receive better prices on NASDAQ, these average comparisons mask systematic differences in NASDAQ and SEAQ dealer discount policies. The middle section of Table 1 shows how the timing of trades can affect how one interprets the differences observed in the top section of Table 1. It reports the percentage distribution of the best bid-ask spread weighted by minutes the markets are open, trades, and trade value. On SEAQ, for example, 26.3 percent of the time (two hours and six minutes of a trading day) a trader can expect to pay a spread of four pence. Roughly one-fourth of SEAQ trades and shares transact at this spread. On NASDAQ, however, the best bid-ask spread is around four pence thirty-nine percent of the time, yet few trades or shares transact at this spread. There are many possible explanations for this phenomenon. NASDAQ traders might be in a better position to trade when spread costs are low, or NASDAQ market makers might compete more for trades during high volume periods. Our general point is that average transaction cost measures do not distinguish between these explanations. Another way of making this point is that these measures do not reveal what the same trade would pay in each market. In what follows, we propose methods that address this question.

Before developing our measures, we first describe features of NASDAQ and SEAQ that influence how we construct and interpret our measures. Figures la-1d display quotation and trading histories for Cadbury on two arbitrarily chosen days, October 16 and 17, 1991. These figures illustrate where trades occurred relative to the best bid and ask 
on SEAQ and NASDAQ. The dark horizontal lines are the best bid and offer. London traders call this the "touch". The dashed vertical lines mark the official open and close on NASDAQ and the unofficial open and close on SEAQ. (Appendix A describes the SEAQ data and trading procedures in greater detail.) Figure la shows the unique information we have on SEAQ trades. It displays customer orders, inter-dealer trades, and crossing trades. Figure $1 b$ displays the NASDAQ data. Like most publically available data, the NASDAQ data do not identify trade counterparties. Figure la shows that the SEAQ touch does not vary much during the course of the trading day. Most customer trades (dots and triangles) go through at the touch. By contrast, many dealer trades (circles and stars) receive execution within the touch. The figure also shows that SEAQ market makers do not use a fixed tick size rule when offering discounts. The black stars represent inter-dealer broker [IDB] trades executed on one of four anonymous electronic bulletin boards. The four IDB systems provide services similar to those offerred to NASDAQ dealers by Instinet. Generally, it appears that SEAQ trades are distributed randomly throughout the day and there are no obvious anomalies when NASDAQ opens. On both days, at least one customer sell order executes outside the touch. These trades seemingly violate SEAQ's best execution rule (See the Data Appendix.).

During this period, sixteen SEAQ market makers and over twenty-five NASDAQ market makers posted quotes and took trades. Though not pictured, each SEAQ market maker had a quoted spread of five pence. That is, the difference between their quoted bid and ask prices was five pence. Cadbury market makers maintained this spread virtually the entire year ${ }^{6}$ Since the market touch was four pence or less on these two days, no SEAQ market maker ever simultaneously posted at the best bid and best ask. Unfortunately, we do not have similar information for NASDAQ. Other studies, however, suggest that NASDAQ market makers are rarely post both the best bid and ask (See Chan, Christie, and Schultz (1993).)

Comparing contemporaneous touch spreads, we see that SEAQ has a slightly larger spread on October 16 and a smaller one on the 17th. These differences are not large enough to cause arbitrage. ${ }^{7}$ Figures $1 c$ and $1 d$ provide information about the size of trades. In each we have scaled the area of the circles to represent the number of shares traded. These figures suggest that in contrast to the NYSE, some but not nearly all large trades execute inside the touch. The IDB trades and broker trades are also larger than the average customer trade. Finally, the figures suggest that large inter-dealer trades usually, though not always, execute within the spread. 


\section{Estimating Transaction Costs - Adjusted Apparent Spreads}

Table 1 and Figures 1a - 1d together reveal substantial differences in transaction spreads. The obvious challange is to devise measures that isolate these differences. Demsetz's (1968) original work suggested one should interpret the quoted spread as a security dealer's price for immediacy. In theory, the price of immediacy is the difference between an investor's purchase or sales price and the asset's "truen or "immediaten value. As Demsetz and others note, researchers and dealers rarely know an asset's true value. There is little agreement among researchers, however, on how to define or measure immediate value. The definition we adopt is the instantaneous cost of a round-trip transaction - what we call the apparent cost or apparent spread incurred by simultaneously purchasing and selling shares. We adopt this definition because it follows Demsetz's concept of immediacy and because it pairs the costs of comparable trades. The obvious practical problem with our definition is that one rarely observes comparable simultaneous buy and sell orders. To understand how we overcome this problem, we briefly summarize other approaches.

Prior researchers have measured transaction costs by averaging best bid-ask spreads or by inferring implicit spreads from neighboring transaction prices. Figure 2a illustrates several problems with these approaches. For simplicity it presumes that the touch is constant. As in Figures $1 c$ and 1d, the areas of the circles represent each trade's size. The effective spread at time $t-1,2 \times E_{t-1}$, measures transaction costs as the deviation of price from the touch midpoint. This measure implicitly assumes that the midpoint is the asset's "true value", or that the discount on "a reverse transaction would receive the same discount. Since on SEAQ the same dealer rarely posts both the best bid and ask, it is unclear why the touch midpoint is the best way to measure a SEAQ security's true value. Indeed, the Cadbury data reveal that the average of dealers' quotes can differ substantially from the touch midpoint. For instance, occasionally fifteen market makers will be at the ask and only one at the bid. Do these positions signal that the touch midpoint is not the "averagen or true price? This question is difficult to answer. However, we would like in principle to have a measure of transaction costs that incorporates this information, since the positions of dealers may affect their willingness to offer discounts.

Figure 2a also displays another popular measure of spread-related transaction costs, those based on differences in neighboring buy and sell transaction prices. At time $t, \Delta P_{t}$ measures the difference between a small buy and a large sell order. This implicit spread 
measure has the advantage that it does not require quotation information. The figure, however, reveals a potential drawback. Since it compares the discounts of different size trades, it can mask size discounts. The same caveat applies to spread measures proposed by Roll (1984) and others. These measures estimate spreads from serial covariances of transaction price changes. Although subsequent research has refined Roll's measure to allow for drifting spreads and dealer inventories, few studies condition these serial covariances on other observable trade characteristics, such as order size. Some studies also have used regression analysis to condition price changes on past price changes and trade characteristics. ${ }^{8}$ These regressions, however, usually do not relate price changes to information about the touch or dealers' quotes.

Figure $2 b$ illustrates how we propose to use trade characteristics and price information to develop a measure of transaction costs. The figure displays a sell order receiving a discount $D_{t}$ from the best quoted bid. We define the apparent spread on this transaction, $A S_{t}$, as the difference between the transaction price $\left(P_{t}\right)$ and the quoted ask. The apparent spread provides an upper bound on transaction costs because SEAQ's best execution would guarantee a reverse purchase execution at or within the ask. An obvious question is, Why is the quoted ask the appropriate benchmark for the reverse (round-trip) transaction? Aside from SEAQ best execution rules, there is no guarantee that a market maker will execute the reverse buy order at the touch.

Since we do not observe the reverse discount, we propose to estimate it. One possible estimate of what a market maker might offer is the discount $D$ on the customer's original sale. If this discount is applied to the ask, we obtain the effective spread. If this discount is applied to a dealer's ask price above the market ask, then we can obtain a price that is outside the touch. This assignment rule therefore can violate the Exchange's best execution rule. Since there is little reason to believe that the same dealer will execute the reverse transaction at the same discount, we propose an econometric model of discounts that uses past information to predict what dealers would offer under current conditions. Using this model, we construct an estimate of the reverse transaction discount $\widehat{D}$ and then define the round-trip transaction cost as the adjusted apparent spread; that is, the difference between the apparent spread and the estimated discount, i.e. $\widehat{A A S}=A S-\widehat{D}$.

The key element of our approach is the econometric model that predicts discounts using trade information. Ideally, we should develop this model from a rich theory that predicts how dealers use information to set spreads and discounts. Formally, we would like 
to know the structure of $D=D\left(\Omega_{t}\right)$, where $\Omega_{t}$ represents the market maker's information at the time of trade. Because we are unlikely to observe everything in $\Omega_{t}$, we must adopt an alternative model. We formulate a conditional prediction model by assuming discounts are random draws from a density

$$
\mathrm{f}\left(D_{t} \mid \omega_{t}\right)
$$

Here $f$ represents the observed density function of discounts and $\omega_{t}$ represents our information. By examining how the observed conditioning variables $\omega_{i}$ affect $f$, we hope to identify what factors determine dealer discounts, and thus transaction costs.

In principle, we could a variety of statistical techniques to estimate how the conditioning variables $\omega_{t}$ affect $f$. We chose to use quantile regressions. These regressions describe how the quantiles (or percentiles) of $D$ vary with $\omega_{l}$. (See Koenker and Basset (1978).). We chose conditional quantiles over means primarily to minimize the influence of misclassified trades, a problem present in most intra-day transaction data sets. To underscore the point that these quantile regressions do not produce "structural" estimates of parameters underlying $D\left(\Omega_{t}\right)$, we suppress in what follows the coefficients from the underlying regressions, favoring instead to report point predictions and approximate standard errors. To the extent that we have statistical hypotheses, they are that particular variables do not explain observed discounts. In work not reported here, we have explored the robustness of our predictions using split sample techniques. These checks convince us that the quantile estimates are reasonably accurate for all but very large trades.

\section{Apparent Spreads for Cadbury \& Schweppes}

To date, we have estimated very simple models of discounts and spreads. In future work, we plan to experiment with other conditioning variables, such as the direction of trades. The specifications we report here examine whether and how discounts vary with the trade counterparties (for SEAQ only), the size of trades, and the touch. While previous studies have examined the separate impact of the touch and the size of trades, our specifications allow for interactions between the two. To our knowledge, we are the first to estimate counterparty effects.

Since we do not use conditioning variables that would generate asymmetric discounts, we treat discounts symmetrically by modelling apparent spreads. We assume SEAQ 
apparent spreads ( $\mathrm{AS}=$ Touch - Discount) equal

(2) $\quad \mathrm{AS}_{i}=$ Touch - Discount $_{i}=\sum_{j=0}^{3}\left[\beta_{0, i}+\beta_{1, i}\right.$ Touch $] \times\left(\text { Trade Size }_{i}\right)^{j}+\epsilon_{i}$

where $i$ indexes types of trades (IDB, customer, market maker and broker trades), the $\beta$ 's are unknown coefficients and $\epsilon$ is an unobserved error. In words, the apparent spreads are a polynomial in trade size and interactions with the touch at the time of the trade. We include touch interactions on the right hand side to allow for the possibility that apparent spreads may depend on the (guaranteed) touch spread. The NASDAQ regressions have a similar structure. They use quartic polynomials in trade size and do not have coefficients that depend on trade types.

Figures 3a-3c plot the estimated apparent spread quartiles by size of trade, the touch, and trader identities. To simplify comparisons between SEAQ and NASDAQ, we have expressed the apparent spreads as a fraction of the prevailing touch. The vertical differences between the top horizontal curves and the horizontal axis equal the estimated apparent spread divided by the touch. The vertical differences between pairs of similarly shaped curves are the estimated adjusted apparent spreads, our measure of the cost of an instantaneous round-trip transaction. Figure 3a displays how the median cost for a SEAQ customer trade depends on the touch and the trade's size. The vertical dashed lines mark the median Cadbury trade size (approximately $£ 4,500$ ) and the largest size trade a market maker must accept at their quoted prices (approximately $£ 100,000$ ). The median size customer trade executes at the touch, no matter what the touch. As Cadbury's touch widens from two to three to four pence, the median large customer trade receives deeper and deeper discounts. At four pence, a trade larger than $£ 1,000,000$ receives roughly a twenty-five percent discount (one pence per share or $£ 2,600$ total). These estimates confirm that only very large (usually institutional) trades are likely to receive discounts. Even these large trades, however, are not assured discounts. The graphs also show that trade discounts do not widen at the same rate as the touch. That is, when the touch widens by one pence, the total discount from the bid and the ask does not increase by one pence. This shows that market makers do not use discounts to maintain a constant pence spread.

Figure $3 b$ summarizes the variation in customer transaction costs holding the touch constant at three pence (the sample median.) The three curves represent the first, second (median), and third apparent spread quartiles. Vertical differences between similar curves 
again equal estimated adjusted apparent spreads. Note that there is no inter-quartile difference in apparent spreads at or below the median trade size. In other words, nearly all small retail orders pay the quoted spread. This finding appears at odds with asymmetric information models that predict small uninformed trades will receive more favorable execution. Upper quartile customer trades appear to receive no discount up to the mandatory quote volume of $£ 100,000$. Surprisingly, beyond the mandatory quote volume the dispersion in discounts increases. Nearly twenty-five percent of customer trades larger than $\mathcal{E} 400,000$ receive at least a fifty percent discount, implying the adjusted apparent spread is zero or negative. The infrequency of large trades, however, reduces the precision of our estimates.

The frequency with which we observe trades of a given type and size is the key determinant of the statistical precision of our estimates. Generally, we observe many more small trades than we do large, and many more trades at three pence than we do two or four pence. A plot of apparent spreads by trade size also reveals that market makers tend to clump discounts in fractional pence ranges, though the Exchange does not have tick size rules. To provide an indication of the precision of our point estimates across ranges of trade values, we calculated standard errors using Chamberlain's (1993) suggested approximations. These estimated standard errors confirm that the (point) precision of our estimates in Figure $3 \mathrm{~b}$ deteriorate as the size of the trade increases. At the median trade size, the standard deviation of the median apparent spread to touch ratio is 0.02 . Thus, at a touch of three pence, a ninety-five percent confidence interval for the median apparent spread is 2.79 to 2.91 pence. For transactions near $£ 150,000$, the standard deviation rises to 0.04 , by $£ 300,000$ it is 0.10 , and by $£ 400,000$ it equals 0.19 . Thus, we do not estimate apparent spreads precisely beyond two to three times the mandatory quote size $(£ 100,000)$.

Figure 3c shows the estimated distribution of spreads for NASDAQ holding the touch constant at three pence. We estimate that more than fifty percent of NASDAQ trades execute at the touch and thus receive no discount. Lower quartile trades receive discounts at most sizes. The NASDAQ quartiles are more curved than SEAQ quartiles, with discounts of fifty percent effectively eliminating the spread for trades over $£ 150,000$. At larger sizes, the discount diminishes and then appears to increase. Since we observe few trades in this range, we do not attach much significance to this increase. For now, we tentatively conclude that NASDAQ and SEAQ market makers have roughly comparable discount policies. 
Finally, Figure 3d displays how the median spreads of IDB, market maker-to-market maker, and dealer-to-dealer (or market maker) trades vary with a trade's size. Our calculations assume the touch is three pence. Because the JEAQ data do not identify which of the two SEAQ dealers initiates a trade, we classify dealer trades as buy (sell) orders based on whether the observed price is above (below) the touch midpoint. The vertical dashed line indicates the mandatory quote volume. Each type of inter-deal trade has a median size roughly equal to the mandatory quote volume. The median market maker-to-market maker trade below $\mathcal{L} 400,000$ pays the full spread. This is perhaps not too surprising since dealers negotiate these trades over the phone and market makers cannot tell whether the order is for the market maker or a customer. When market makers deal anonymously with each other using inter-dealer brokers, however, they discount the spread by about one-third, or one pence. This also is not surprising since IDB users purchase for (sell from) their own account so that they can subsequently sell to (buy from) a customer. SEAQ brokers seem to grant market makers and other brokers deep discounts. The median broker discount reaches a maximum of more than than one-third the touch, or one pence, for trades around $\mathfrak{\$ 4} 50,000$. Curiously, we observe few broker trades compared to the number of market maker-to-market maker trades.

\section{Inter-Security Variations in Quoted and Touch Spreads}

The Cadbury example suggests that transaction costs vary systematically with trader identities and order sizes. This naturally leads us to question whether the Cadbury example is representative of SEAQ transaction costs. Ideally we would like to answer this question by using quantile regressions to estimate which factors affect each SEAQ security's adjusted apparent spread. These calculations would allow us to distinguish between security, security class, and exchange specific variations in transaction costs. Unfortunately, our data and econometric methods currently do not allow us to analyze a large sample of SEAQ securities. The main obstacle we face is the time required to match separate transaction and quotation records. The Exchange transactions records, for example, require extensive checking to identify IDB trades and to match "shape" trades. (See the Data Appendix.)

Although we continue to work toward a longer term goal of matching all SEAQ quotation and transaction data, this paper examines two narrower SEAQ samples. This section analyzes the fourth quarter 1991 quotation records of 1,887 UK and Irish equities. 
The main advantage of this sample is its broad coverage. These securities accounted for over 95 percent of 1991 SEAQ trading volume. Its main drawback is it only contains quotation information. Consequently, we can only make statements about how quoted prices and volumes vary across securities. The next section use matched quotation and transaction data on sixty of these securities to find whether customers pay quoted spreads.

\section{The Relation Between Spreads and Dealer Concentration.}

The Cadbury results suggest that we should find differences in spreads across securities, if only because the size of trades and characteristics of traders will vary across securities. The main issue we address here is, are there other factors that may cause residual differences. We can think of several, including the inherent riskiness of securities, the amount of total trading volume, and the number and identities of market makers.

Previous studies of NASDAQ spreads have found significant cross-section variation in best bid-ask spreads. Much of this variation appears related to trading volume, with spreads declining rapidly as share volume increases and dealer concentration decreases. Some researchers interpret these relations as evidence of the benefits of market maker competition. That is, market makers compete harder when there are more market makers. Other researchers attribute the decline to the high cost of marketing low volume equities. What is unclear is why do dealers of low volume stocks have high costs? Given the similarities in the SEAQ and NASDAQ quotation systems, we also might expect to see spreads on SEAQ securities fall as trading volume increases. Indeed, we do. This raises the issue, how should one interpret the rate at which spreads decline? Our answer is that the decline reveals the economies of scale in market making.

Several rules introduced by the Exchange in the mid-eighties encourage market maker competition on SEAQ. First, the Exchange allows free entry into market making provided market makers have adequate capital. Second, the Exchange also allows market makers to quit or add securities on short notice. Third, the major costs of making markets, the market makers' time and capital, are largely fixed and not sunk costs. Together, these conditions suggest that even market makers for small volume stocks face substantial (potential) competition. Provided there is some slope to the demand for any individual market maker's services, this competition will result in a familiar monopolistic competi- 
tion equilibrium: competitive entry will make each market maker's demand curve tangent to their average dealing cost curve. If trading volume in a stock increases, competing market makers will enter, and reestablish the tangency condition. Afterwards, each market maker will operate at a higher volume and charge a lower spread (since average dealing costs decline with volume). Thus, in a monopolistically competitive dealer market, the fall in spreads with trading volume reveals the shape of market makers' average dealing cost function and the extent of scale economies. (See Bresnahan and Reiss (1991).) Also, this theory predicts that it is the number of actual and not potential competitors that best predicts the decline in spreads. For example, if the number of market makers that could potentially make a market increases from two to ten, we would see no change in spreads. Whereas, if the ten entered, we would see a decrease in spreads.

Table 2 and Figures $4 \mathrm{a}$ and $4 \mathrm{~b}$ report how the distribution of quoted spreads and best bid-ask spreads vary across the 1,887 SEAQ equities. In each, we condition spreads on the number of market makers posting quotes, as opposed to the number of market makers eligible to post quotes. We note again that this conditioning does not have a causal interpretation. Instead, we base this conditioning on the monopolistic competition prediclion that securities with little trading volume will have few dealers, and that each of these dealers will have higher costs. The spreads underlying the table and figure are quarterly medians of average daily spreads. We calculated a security's average daily spread by averaging minute-to-minute spreads during the mandatory quotation period: 8:30 a.m. to $4: 30$ p.m.. To summarize the variation in these median spreads given a particular number of quoting market makers (i.ei market structure), we report the quartiles of these medians. Thus, the median of the median touch spreads of monopoly dealers is 14.83 percent of price. The median touch spread in the highest six monopoly markets is greater than 28.57 percent of price, and so on. Table 3 shows that the difference in the median touch spreads for markets with one versus five market makers is twelve percent of price. The inter-quartile range for five market makers is only two percent of price. This large drop suggests there are scale economies at small volumes, and that low volume dealers have excess capacity. The median mandatory quote volumes in the far right column, which are based on the past twelve months' trading volume, confirm that customer transaction costs, as measured by touch spreads, fall with trading volume.

Figure $4 \mathrm{a}$ graphically displays how quickly the inter-quartile range and medians of quoted spreads fall as market concentration decreases and share volume increases. Figure $4 \mathrm{~b}$ does the same for touch spreads. The figures and tables suggest that dealers achieve 
most scale economies in markets with mandatory quote volumes of $£ 15,000$ to $£ 25,000$, which roughly corresponds to a market with five to eight dealers. Column 9 of Table 2 provides additional supporting evidence. It shows that the median cost of a 1,000 share trade executed at the touch falls from $£ 57.28$ when trading with a monopolist to around $£ 16$ - $£ 20$ when trading with a dealer with four to seven competitors. The quoted spreads of the largest stocks in our sample, the FTSE-100 equities, exhibit little inter-quartile dispersion, conditional or unconditional on the number of market makers. A monopolistic competition model would predict that these dealers are near the bottom of their average dealing cost curves, which roughly corrsponds to mandatory quote volumes of $£ 50,000$ to $£ 75,000$. Based on the Exchange's rule for calculating mandatory quote volumes, this corresponds to an annual trading volume of between $£ 500$ to $£ 700$ million.

The figures and Table 2 reveal other interesting regularities. Touch spreads fall at roughly the same rate as quoted spreads. In percentage terms, the gap between the two widens as the number of market makers increases. This perhaps suggests that market makers allow themselves more leeway in moving quotes when they face greater competition. The relation is still somewhat odd, since there is no obvious reason why quoted (as opposed to equilibrium spreads) should change with the number of competitors. Table 2 also reveals how dealers' posting behavior may affect the difference between quoted and touch spreads. Columns ten through twelve of Table 2 show that market makers are usually either at the best bid, best ask or setting quotes outside the market. For instance, the median security with nine market makers will have four market makers at the best bid and four at the best ask. The gap between the touch and quoted spread suggests the four dealers setting the best ask are not the same four setting the best bid. The remaining market maker straddles both the best bid and ask. By doing so, this market maker avoids most of the inventory and information risks associated with unsolicited trades.

The second to last column of Table 2 reports the median number of times per day a market maker changes their price quotes. Although we know of no obvious benchmark for this number, we were struck by how infrequently market makers adjust their stated willingness to take trades. One might attribute constant quoted prices to infrequent turnover, yet even FTSE-100 market makers change their quotes less than once per hour! One explanation for these persistent prices is that ihe large mandatory quote volumes substantially increase market makers' capital risk. This risk causes them to widen spreads. By fixing wide spreads, they retain the option to vary transaction prices 
without changing quotes. We now consider how frequently they offer discounts from the touch spread.

\section{Further Evidence on Apparent Spreads}

The analyses in sections 2 and 3 reveal substantial variation in spreads by security, trader, time, and trade size. This section estimates an econometric model that isolates the contribution of these factors to apparent spreads. Our data sample consists of fourth quarter 1991 quotation and transaction histories for sixty SEAQ securities. We randomly chose the sixty securities so that they would equally represent large (i.e. FTSE-100), medium and small capitalization SEAQ securities. We limited the sample to sixty securities because of the time required to match and check quotation and trade data. The fourth quarter is the most recent we have. (See Appendix B.)

The three market capitalization classes roughly divide the sixty securities into three volume, market structure and price size classes. Tables 3 and Al provide information on these classes and the sample securities. Comparing Tables 2 and 3, it appears that the sample represents the range of SEAQ dealer concentration and trading volumes. During the mandatory quotation period, the FTSE-100 securities averaged 106 trades amounting to $£ 7,156,000$, compared to 13 trades of $£ 646,000$ for medium, and 5 trades totalling $£ 83,000$ for smaller equities. The average number of market makers ranges from 12.6 for FTSE-100 equities, to 6.2 and 4.7 for medium and small equities. The quoted and touch spread statistics in Table 3 also span those in Table 2.

Table 3 provides median quoted, touch, effective and adjusted apparent spreads for each security. Although these medians mask intra-security variation in transaction costs, they reveal considerable variation in spreads within and across size-classes. Most of this inter-security variation occurs because these securities have different security prices, and not because they have different pence spreads. For instance, Cadbury \& Schweppes (CBRY) has median touch, apparent and effective spreads of three pence. So does Abbey National. The percentage differences in Table 3 occur because Abbey's price is two-thirds Cadbury's price. Although on other exchanges the fixed spread might occur because of tick size rules, the LSE does not regulate tick sizes. Thus, it is puzzling that absolute SEAQ spreads do not vary more with price. We also observe that the variation here is similar, though perhaps more substantial, than that reported for NASDAQ firms by Stoll (1989). He reports spreads for NASDAQ/NMS stock size deciles. They range from 1.2 
to 6.9 percent of price. A recent study by Chan, Christie, and Schultz (1993) using a more recent time period reports higher average percentage spreads for large NASDAQ stocks. $^{9}$

The within-class variation in spreads and discounts in Table 3 does not appear related to dealer concentration or volume. Compare, for example, the two oil companies, Richmond Oil and Gas (ROG) and Crossroads Oil (COI). They have the same touch and the same number of market makers. Richmond, however, has a much greater median discount. The greater discount granted the median Richmond trade may reflect Richmond's larger average trade size. Yet, if trade size explains this difference, then why do we not observe greater differences between Richmond and other securities?

As suggested by the analyses in Sections 2 and 3, the inter-class differences in spreads might reflect differences in trade counterparties and the volume of trade. Table 4 reports information on the distribution of trade counterparties and trade sizes for each of the three size classes. Most trades in each class are "customer bargains"; that is, trades where a retail customer is a counterparty. Market makers execute between sixty and seventy-five percent of these trades. This is somewhat surprising since the Exchange has more than 300 brokers and just 27 market makers. When brokers do trade with customers, they typically execute large transactions. Relative to other trades, we rarely observe agency crosses, or customer-to-customer trades. Curiously, these trades occur more frequently among smaller (less liquid) issues. The average FTSE-100 equity has 106 customer trades per day, which vastly exceeds the number of customer trades for medium (13) and small (5) equities. Although the number of trades per day differs substantially, the average and median trade sizes do not. ${ }^{10}$ Thus, this table suggests that average trade sizes alone do not explain differences in median spreads.

The next section of Table 4 examines whether inter-dealer trade discounts differ. If particular types of inter-dealer trades receive large discounts, then we might expect this to affect average spreads. As a group, the FTSE-100 sample equities have considerably more inter-dealer trades than either the medium or small equities. Table 3, however, shows that the smaller equities receive greater percentage discounts. Thus, the total volume of inter-dealer trades does not obviously appear related to discounts. Table 4, however, also shows that the four inter-dealer broker systems account for an appreciable fraction of total inter-dealer trades. This suggests that market makers can take advantage of discounts offered on electronic systems that are not offered over the phone. Together, 
these results suggest that while differences in the mix of customer and inter-dealer trades can explain some variation in spreads and discounts, they are not the only sources of variation.

Table 5 describes how median apparent spreads and discounts differ with trade counterparties. To facilitate comparison, it contains apparent spreads and discounts expressed as fractions of the prevailing touch. No adjustment is made for differences in the size of trades or trading volume. As in the Cadbury example, the median trade between a customer and a nuarket maker receives no discount. The median customer trade through a broker does receive a discount. These discounts are small for FTSE-100 securities and large (one-quarter to one-third of the touch) for medium and small size class securities. Customer crosses receive large discounts. This is not too surprising since these trades usually involve large institutions swapping equity "baskets." Median inter-dealer trades receive modest discounts, more so in the medium and smaller equity size classes. Most IDB trades and trades between dealers occur at or close to touch midpoints. " Infrequent dealer crosses usually occur at one-half the touch. The table also shows asymmetries exist. For example, the median discount on a medium class customer buy from a dealer is twenty-five percent of the touch. The corresponding discount for a customer sell is eight percent. This asymmetry reinforces our earlier point that dealer discounts may have little to do with the touch midpoint.

\section{Quantile Regression Results}

Following the Cadbury \& Schweppes analysis, we can use quantile regressions to decompose apparent spreads for these sixty securities into volume, trader and securityspecific components. In principle, we could proceed by estimating separate regressions for each security. In practice, this approach is computationally and descriptively unweildy. We instead estimated separate models for each size class. These specifications include security fixed effects. Additionally, because spreads differ across securities in relation to the security's price, we scaled the apparent and touch spreads by security prices. Experimentation with functional forms led us to the following specification describing customer trades for security $j$

(3) $\frac{\mathrm{AS}_{j}}{P_{j}}=\beta_{0}+\frac{\text { Touch }_{j}}{P_{j}} \times\left[\theta_{j}+\beta_{00} \frac{\text { Touch }_{j}}{P_{j}}+\sum_{i=1}^{4}\left[\beta_{i}+\beta_{0 i} \frac{\text { Touch }_{j}}{P_{j}}\right](\text { Trade Size })^{i}\right]+\epsilon$

In words, we represent the conditional quantiles of apparent spreads as a quartic in the trade's pound value interacted with the touch and the square of the touch. The 
coefficient $\beta_{0}$ is the apparent spread when the touch is zero. The coefficient $\theta_{j}$ is a security-specific fixed effect that captures differences in discounts from the touch across securities. The polynomial in size and the squared touch give added flexibility to the shape of the conditional median discount function. To capture differences in customer and non-customer trades, we included additional zero-one dummy variables interacted with the touch and the touch squared when the trade involved an IDB, a dealer, or two market makers.

Table 6 summarizes the results of the conditional median regressions for each size class. The top left panel describes the fit. While there is no natural measure of fit, the average absolute error is small and the conditional medians explain about sixty percent of the variation in apparent spreads (over price). Figure 5 plots actual versus fitted values for the medium size class. It shows that the model does reasonably well explaining the substantial variation in apparent spreads. The security specific fixed effects explain little of the variation in apparent spreads. In future work, we plan to investigate whether there are more complicated security-specific size and trader effects. The other three panels in Table 6 suggest that the model explains a large fraction of the variation across securities, but also that there are exceptions. The inter-quartile range statistics (columns (3) and (6)) show that trade size, touch and trade type explain variation in apparent spreads. The large absolute errors and standard deviations show, however, that there are outlying spreads the model does not explain. We do not have an explanation for these unusual spreads, although most occur because of trades outside the quoted spread. These observations may represent match or coding errors.

Figures $6 \mathrm{a}$ to $6 \mathrm{c}$ display estimated conditional median apparent spreads for customer trades by size class. The vertical distance between the upper curves and the horizontal axis is the estimated apparent spread. As in the Cadbury example, the vertical distance between similar curves equals the adjusted apparent spread. Each median is evaluated at the average of the firm effects. Figure 6a plots median adjusted apparent spreads for FTSE-100 equities as the touch ranges from 0.5 to 1.5 percent of price. These estimates are similar to those for Cadbury. Medium and large trades $(£ 75,000-500,000)$ receive only slight discounts when the touch is 0.5 percent of price. As the touch widens, the median FTSE-100 trade obtains a greater discount.

Figures $6 \mathrm{~b}$ and $6 \mathrm{c}$ repeat the format of Figure $6 \mathrm{a}$ for medium and small size equities. The sample average touches are 1.3 percent of price for the medium size class and 2.3 
percent of price for the small class. For the medium size class, median spreads fall monotonically as the size of the trade increases. The same is true for the small size class, but the decline is less pronounced. For instance, when the touch is one percent of price, trades greater than $\mathcal{£} 4,500$ receive virtually no discount. Table 3 shows that a one percent touch-to-price ratio is at the low end of medium and small size class spreads. he median FTSE-100 trade receives a greater discount the wider the touch, although for the very largest trades (not pictured) there is evidence that suc discounts disappear. These differences in discounts across size classes suggest that large orders have either very different competitive or cost consequences for market makers.

We finally display the estimated median discounts for dealer trades in FTSE-100 securities holding the touch constant at one percent of price. Again, these results parallel those in the Cadbury example. Dealer trades have a profile similar to customer discounts, though the discount levels differ. Market makers do not discount the median trade. Brokers grant each other approximately one-third discounts and IDB trades execute at roughly the touch midpoint. Translated into adjusted apparent spreads and not adjusting for the direction of trades, this corresponds to a one-third broker-to-broker spread and a zero IDB spread.

\section{Conclusions}

This study used unique SEAQ transaction and quotation data to document SEAQ spreads and market maker discounts. To the best of our knowledge, this is the first study to account simultaneously for differences by type of trade, trade volume and security. We began by first developing a new measure of transaction costs, the adjusted apparent spread. This measure incorporates information on dealers' quotes and investors' trade characteristics to calculate the hypothetical cost of an (immediate) round-trip transaction. We used quantile regressions to model how these spreads varied with trade, trader and security characteristics. Our estimates reveal that medium to large trades on average receive discounts from the touch spread. These discounts increase the wider the touch. Small and very large trades pay the touch (and sometimes more). Dealers and market makers price customer trades differently. Market makers only discount very large trades; dealers regularly discount medium and large trades. Market inakers rarely discount trades with other market makers over the phone, but do do so when trading anonymously using IDBs. 
The practice of discounting the touch raises many fascinating questions which need further study. We would like to develop theoretical models that explain why dealers grant discounts and how discounts affect spreads. We also would like to understand why dealers link these discounts to size. The pattern we observe suggests that neither simple asymmetric information nor inventory risk models can easily explain why dealers widen spreads and then selectively discount. The anonymous role of IDBs in inter-dealer trades also deserves further study. Finally, it would be useful to develop a monopolistic competition model of market making that recognizes market makers spread their dealing costs across different securities.

Our empirical analysis is preliminary and leaves many issues untouched or partially addressed. We clearly should estimate adjusted apparent spreads using information about who orginates trades. We also need to extend the data samples to explore whether dealers individually have different discount policies. Other market maker information also should enter our regressions. For instance, although we condition on the touch at the time of trade, we do not control for market depth at the bid and the ask, or past information. Additional variables might include market indicies, and capital or price risk measures.

Finally, our findings suggest that researchers should not use average spreads to measure the efficiency of a market. Instead, one should compare measures that hold constant characteristics that market makers "price." While our research does not provide an analytical understanding of how dealers decide on quoted and transaction prices, it provides a place to begin. 
Data Appendix

\section{A. Quotation and Transaction Data}

The Cadbury \& Schweppes NASDAQ transaction data come from the Institute for the Study of Securities Markets [ISSM]. On NASDAQ, Cadbury \& Schweppes trades as American Depository Receipts [ADRs]. We converted the NASDAQ shares to poundequivalent shares using daily FT-Actuaries prices quoted in London.

The SEAQ data come from the Quality of Markets Group [QMG] at the London Stock Exchange. Both the SEAQ transactions and quotations data required extensive editing to match samples and dealer codes. For the Cadbury analysis, we use all overlapping trade and quotation data. These data cover January 14 to March 18, April 2 to June 24, July 1 to September 24, and October 14 to December 31. In sections 3 and 4 we use October 14 to December 31. The quotation data cover 2,000 U.K. and Irish ordinary shares. Some securities have missing data because of retrieval problems, new listings, delistings, or trading halts. SEAQ rules require market makers to quote guaranteed prices and volumes between $8: 30 \mathrm{am}$ and $4: 30 \mathrm{pm}$ London time. Dealers sometimes post prices for up to one-half hour before the mandatory open and close. These quotes are not binding. During the mandatory quote period, market makers must accept trades as large as 2.5 percent of the security's previous twelve month's average daily trading volume. Dealers must also offer "best execution." During 1991, this meant SEAQ market makers had to execute trades less than the mandatory quote volume at or inside the prevailing touch. Market makers away from the touch had to execute their orders at the touch price (or better) or refuse it.

We obtained transaction data for 907 larger U.K. and Irish securities. Approximately 840 of these equities appear in the quotation sample. The transaction data come from end-of-day settlement reports filed with the Central Checking Section of the Stock Exchange. These reports do not necessarily reproduce the original ticker tape. Each trade has two time stamps, one reported by the seller and one by the buyer. We use the seller's time stamp unless it indicates a trade outside the prevailing touch. If the seller's time stamp would classify the trade as outside the touch, we check the buyer's time stamp. If the buyer's time stamp puts the trade at or within the touch, we use the buyer's time. If neither time stamp appears valid, we use the seller's time. This reduces the number of trades that execute outside the touch, but does not eliminate them. Trades outside 
the touch are sometimes "average price" basket trades. Dealers execute baskets using prearranged pricing formulas. The data also contain coding anomalies. The most significant are "shapes." Shapes occur when a dealer matches several customer orders with one (sometimes two) other customer order(s). These appear in the data as a series of unbalanced customer transactions.

\section{B. Pilot Sample with Quotes and Transactions}

We constructed the sample of sixty securities by randomly sampling names from a list of all SEAQ equities. We first assigned securities on this list to market capitalization classes based on their March 31, 1991 market capitalization. ${ }^{1}$ We then randomly sampled within classes, rejecting any security lacking complete data. We also required that it have a mandatory quote size of at least 2000 shares and more than 700 trades between March 31, 1990 to March 31, 1991. We chose the twenty FTSE-100 firms so that they would overlap with previous London Stock Exchange studies.

Table Al provides additional information on the sixty firms. 


\section{Endnotes (Text)}

1. Quality of Markets Review - Summer 1991, pp. 17-24.

2. Some of these predictions carry over to models of dealer markets. For papers that model inventory risks, see e.g. Garman (1976), Amihud and Mendelson (1980), and Ho and Stoll $(1981,1983)$. Admati (1991) surveys papers that model adverse selection risks.

3. Lee (1993) finds liquidity premia, defined as an absolute difference between trade prices and the bid-ask midpoints, that increase with trade size.

4. See the Stock Exchange Quarterly and Quality of Markets Review, Spring Edition $(1992 ; 27)$.

5. SEAQ does not require market makers to offer best execution to very large trades. For additional evidence on SEAQ size discounts see Breedon (1993), de Jong, $\mathrm{Nij}$ man, and Röell (1993), and Hansch and Neuberger (1993).

6. There are a few instances when market makers posted wider spreads (seven, eight, and ten pence) but these are extremely rare.

7. Lang and Werner (1993) discuss costs associated with the arbitrage of ADRs.

8. Compare Ho and Macris (1984), Glosten (1987), Glosten and Harris (1988), Stoll (1989), Harris (1990), and Madhavan and Smidt (1991), Hasbrouck (1991), Lee and Ready (1991) and Lee, Mucklow and Ready (1993). See also Harris's (1990) survey.

9. Several studies report lower spreads for NYSE stocks. For instance, Kleidon and Werner (1993) report an average quoted spread of 0.6 percent for S\&P 100 stocks in 1991.

10. Median trade sizes (not reported in Table 3) are: FTSE-100 $£ 3,744$; medium size $£ 4,550$; and small equities $£ 2,625$.

11. The negative adjusted apparent spreads occur because we separately estimate median spreads and discounts. 


\section{Endnotes (Appendix)}

1. Quality of Markets Companies Book 1991, Table 1: "1000 largest listed UK companies by market valuation," p. 19-38. 


\section{References}

Admati, A., 1991, The Information Role of Prices: A Review Essay, Journal of Monetary Economics, 48 (3), 347-361.

Amihud, Y. and H. Mendelson, 1980, Dealership Markets: Market-Making with Inventory, Journal of Financial Economics 8, 31-53.

Biais, B., 1993, Price Formation and Equilibrium Liquidity in Fragmented and Centralized Markets, Journal of Finance 48, 157-85.

Breedon, F.J., 1993, Intraday Price Formation on the London Stock Exchange, LSE Financial Markets Group Discussion Paper No. 158.

Bresnahan, T.F., and P.C. Reiss, 1991, Entry and Competition in Concentrated Markets, Journal of Political Economy, 99, 977-1009.

Brock, W.A., and A.W. Kleidon, 1992, Periodic Market Closure and Trading Volume: A Model of Intraday Bids and Asks, Journal of Economic Dynamics and Control 16, 451-89.

Chamberlain, G., 1993, Quantile Regression, Censoring, and the Structure of Wages, (Economics Department, Harvard University).

Chan, K.C., Christie, W.G., and P.H. Schultz, 1993, Market Structure and the Intraday Evolution of Bid-Ask Spreads for NASDAQ Securities, (College of Business, Ohio State University).

De Jong, F., Nijman, T. and A. Röell, 1993, A Comparison of the Cost of Trading French Shares on the Paris Bourse and on Seaq International, LSE Financial Markets Group Discussion Paper No. 169.

Demsetz, H., 1968, The Cost of Transacting, Quarterly Journal of Economics 82, 33-53.

Dennert, J, 1993, Price Competition between Market Makers, Review of Economic Studies $60,735-51$.

Foster, F.D., and S. Viswanathan, 1993, Variations in Trading Volume, Return Volatility, and Trading Costs: Evidence on Recent Price Formation Models, Journal of Finance 48, 187-211. 
Garman, M., 1976, Market Microstructure, Journal of Financial Economics 3, 257-75.

Glosten, L.R., 1987, Components of the Bid-Ask Spread and the Statistical Properties of Transactions Prices, Journal of Finance 42, 1293-307.

Glosten, L.R., 1989, Insider Trading, Liquidity, and the Role of the Monopolist Specialist, Journal of Business 62, 211-35.

Glosten, L.R., 1992, Equilibrium in an Electronic Open Limit Order Book, Graduate School of Business - Columbia University Working Paper No. FB-92-93.

Glosten, L.R., 1993, Intra- and Inter-Market Competition, (Graduate School of Business, Columbia University).

Glosten, L.R. and L. Harris, 1988, Estimating the Components of the Bid-Ask Spread, Journal of Financial Economics 21, 123-42.

Grossman, S.J., and M.H. Miller, 1988, Liquidity and Market Structure, Journal of Finance 43, 617-37.

Hansch, O., Naik, N. and S. Viswanathan, 1993, Market Making on the London Stock Exchange: An Empirical Investigation, (London Business School).

Hansch, O., and A. Neuberger, 1993, Block Trading on the London Stock Exchange, (London Business School).

Harris, L.E., 1990, Liquidity, Trading Rules, and Electronic Trading Systems, New York University Salomon Center, Monograp Series in Finance and Economics 1990-4.

Hasbrouck, J., 1991, Measuring the Information Content of Stock Trades, Journal of Finance 46, 179-207

Ho, T, and R. Macris, 1984, Dealer Bid-Ask Quotes and Transaction Prices: An Empirical Study of Some AMEX Options, Journal of Finance 39, 23-45.

Ho, T. and H. Stoll, 1981, Optimal Dealer Pricing Under Transactions and Return Uncertainty, Journal of Financial Economics 9, 47-73.

Ho, T. and H. Stoll, 1983, The Dynamics of Dealer Markets Under Competition, Journal of Finance 38, 1053-74. 
International Organization of Securities Commissions, 1993, Transparency on Secondary Markets: A Synthesis of the IOSCO Debate, Ore Societa Editoriale Media Economici, Milano, Italy.

Kleidon, A.W., and I.M. Werner, 1993, Round-the-clock Trading: Evidence for U.K. Cross-Listed Securities, NBER Working Paper No. 4410.

Koeneker, R. and G. Bassett, 1978, Regression Quantiles, Econometrica 46, 33-50.

Lang, M., and I.M. Werner, 1992, U.K. Cross-Listed Securities: Who Sets the Price?, (Graduate School of Business, Stanford University).

Lee, C.M.C., 1993, Market Integration and Price Execution for NYSE-listed Securities, Journal of Finance, 1009-1038.

Lee, C.M.C, Mucklow, B., and M.J. Ready, 1993, Spreads, Depths, and the Impact of Earnings Information: An Intraday Analysis, Review of Financial Studies 6, 345-374.

Lee, C.M.C., and M.J. Ready, 1991, Inferring Trade Direction from Intraday Data, Journal of Finance 46, 733-746.

Madhavan, A., 1992, Trading mechanisms in Securities Markets, Journal of Finance 47, 607-641.

Madhavan, A., and S. Smidt, 1991, A Bayesian Model of Intraday Specialist Pricing, Journal of Financial Economics 30, 99-134.

McInish, T., and R. Wood, 1992, An Analysis of Intraday Patterns in Bid/Ask Spreads for NYSE Stocks, Journal of Finance 47, 753-64.

Neuberger, A.J., 1992, An Empirical Examination of Market Maker Profits on the London Stock Exchange, Journal of Financial Services Research, 343-372.

Neuberger, A.J., and R.A. Schwartz, 1989, Current Developments in The London Equity Market, (London Business School).

Reinganum, M.R., 1990, Market Microstructure and Asset Pricing - An Empirical Investigation of NYSE and NASDAQ Securities, Journal of Financial Economics 28, 127-147. 
Roll, R., 1984, A Simple Measure of the Effective Bid/Ask Spread in an Efficient Market, Journal of Finance 39, 1127-39.

Stoll, H.R., 1989, Inferring the Components of the Bid-Ask Spread: Theory and Empirical Results, Journal of Finance 44, 115-134.

Stoll, H.R., 1992, Principles of Trading Market Structure, Journal of Financial Services Research 6, 75-107. 


\section{List of Tables}

Table 1: SEAQ and NASDAQ Descriptive Statistics for Cadbury \& Schweppes.

Table 2: SEAQ Quote Statistics by Number of Market Makers.

Table 3: SEAQ Median Spreads as a Percentage of Average Trade Price

Table 4: SEAQ Trading Volume by Type of Trade

Table 5: SEAQ Median Apparent Spreads and Discounts by Type of Trade (Percent of Touch)

Table 6: Summary of Apparent Spread Quantile Regresssions

Table A1: Descriptive Statistics for Transactions Sample 
Table 1 SEAO and NASDAO Descriptlve Stallatlea tor Cadbury \& Schweppes

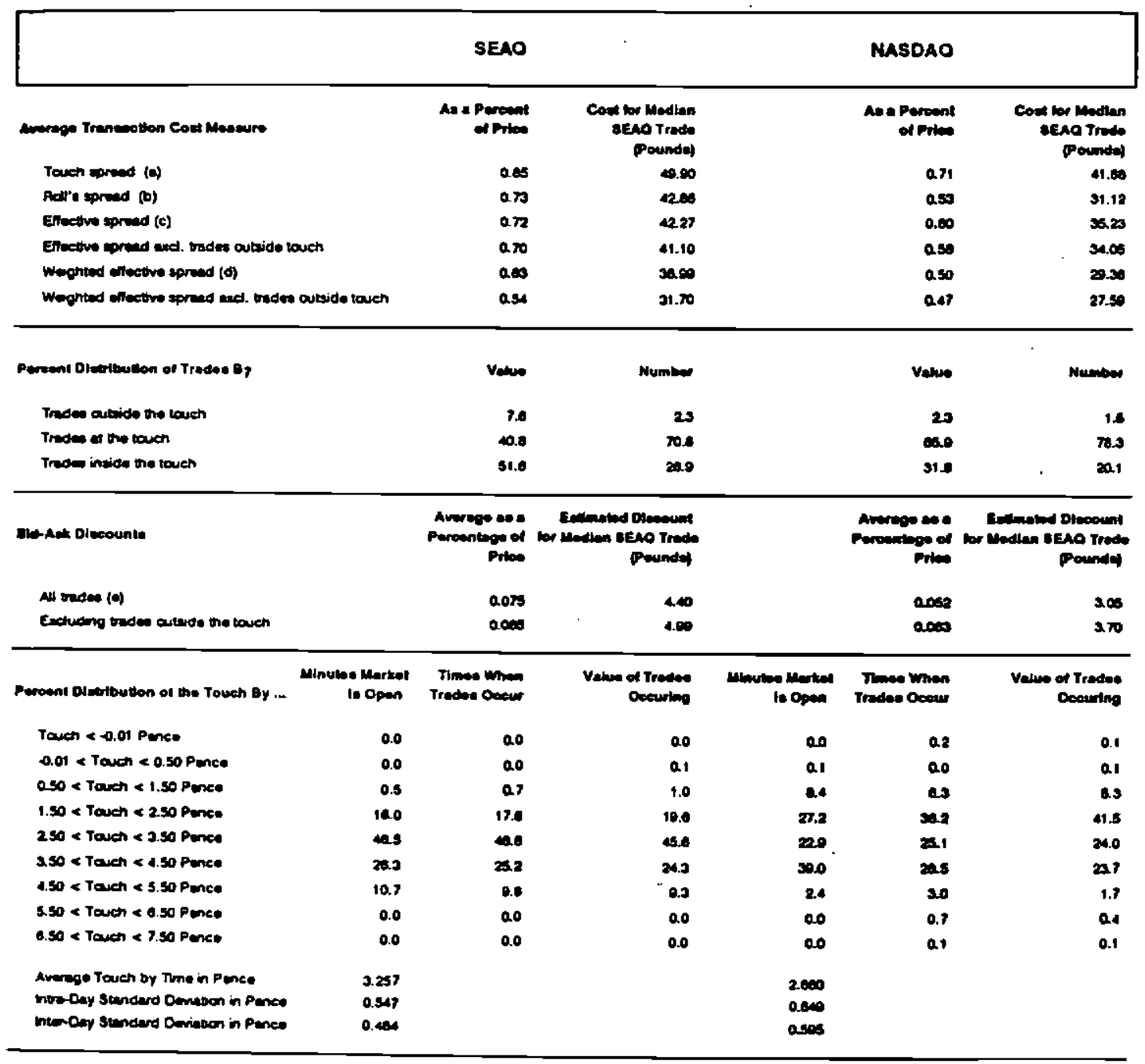

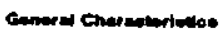

Averege bansectoen pres in pande

Number of meriet makere

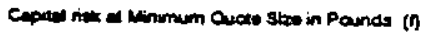

Number of ind

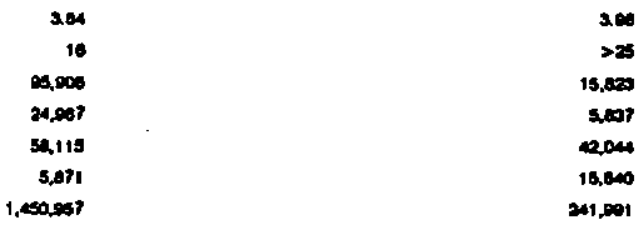

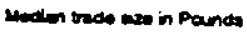

Tow trach vetume in 1,000 Pands 
Sources: SEAO data were drawn from the LSE computer records for the periods January 14 - March 18 , April 2 - June 24. July 1 - Seplember 24, October 14 - Decamber 27. 1891. The miasing parlods are due to ratrieval problems. NASDAO dala were drawn trom the ISSM tapee lor January 1 - Docember 31, 1991.

All statistics oxclude trades belore 8:30 (8:00) am and atter 4:30 (4:00) pm for SEAO (NASOAO). Notes:

(a) The touch spread is the everage across tranactions of $100^{*}$ (Ask-Bld)/Tredo Price.

(b) Roll' spread measure in hwo times the equare root of minus the cerial covariance of price changes.

(c) The effective epread is the average ecrose transections of 2*100*| Trade Prica-(Best Ask+Best Bld)/2|/Trado Prico

(d) The volume-wrolghted average of (c). See Lee and Roady (1991).

(o) The average discount la the average acrose tranactions of 100-1Trade Price-Best Quote / Trade Price

where the Best Quote is the Bid (Ask) lor a customer rell (buy).

(i) Capital itsk is calculated based on the average slock price. The minimurn quote alze for Cadbury $\&$ Schweppes is 25,000 shares on SEAO. Excoptions are given for two market making firms who may poat smaller quote sizes. The maximum quote size lor NASOAO trading of Cadbury \& Soweppes was 1,000 ADRa. 


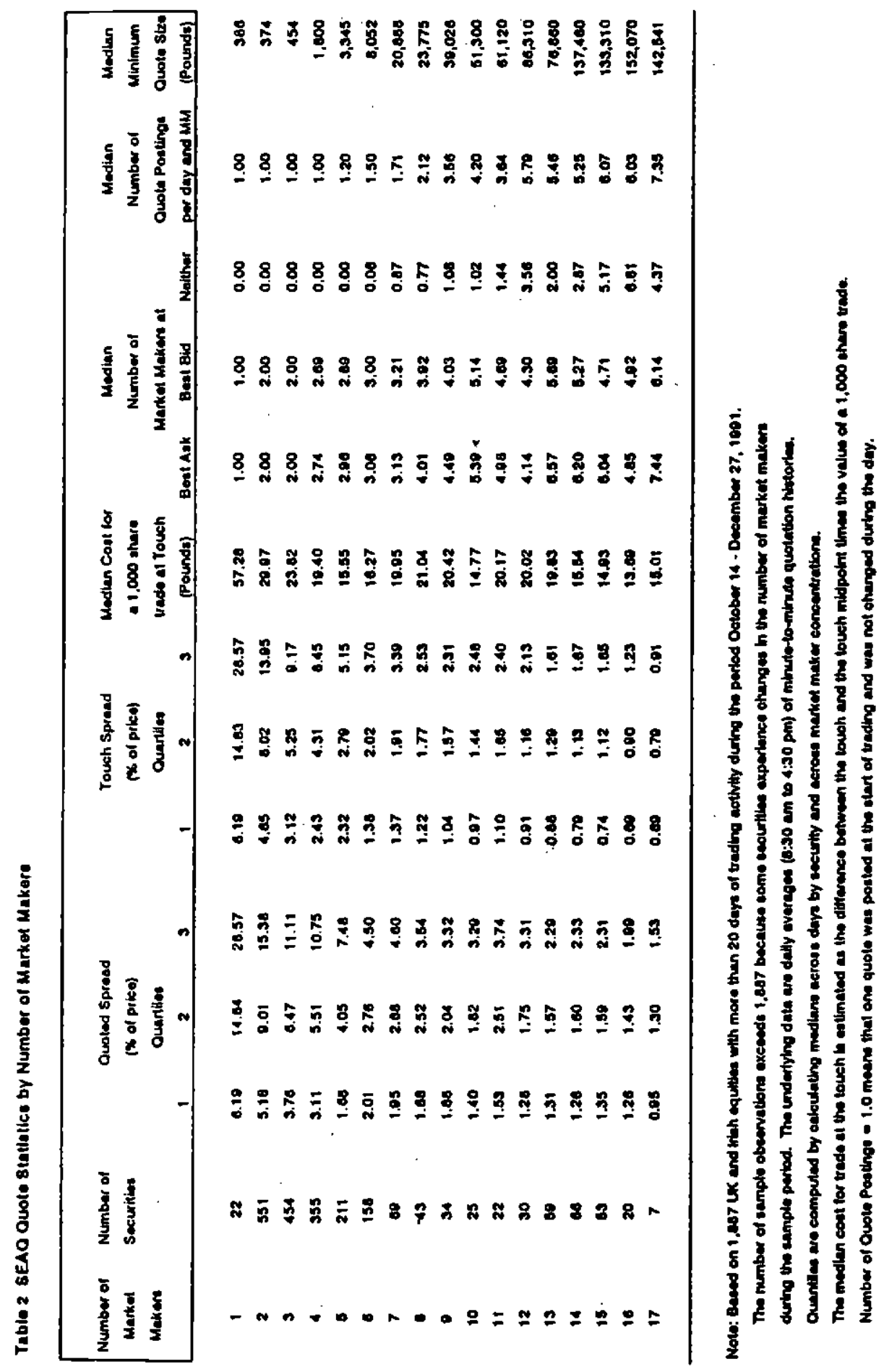




\begin{tabular}{|c|c|}
\hline 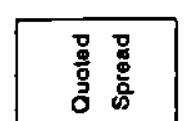 & 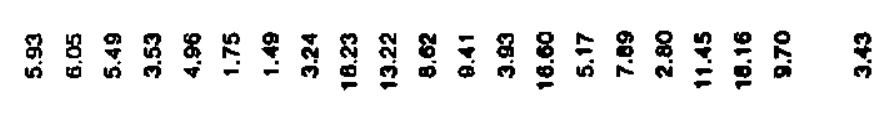 \\
\hline $\mid$ & 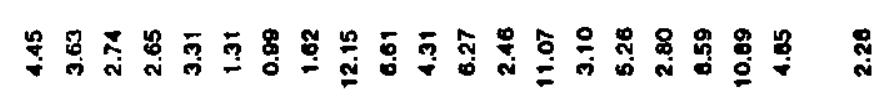 \\
\hline 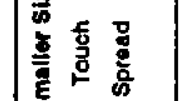 & 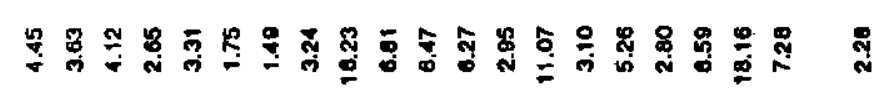 \\
\hline 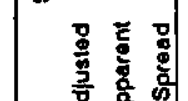 & 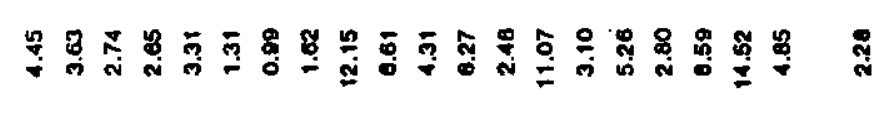 \\
\hline 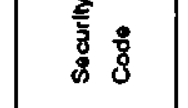 & 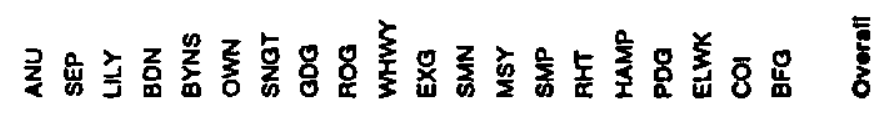 \\
\hline 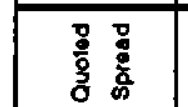 & 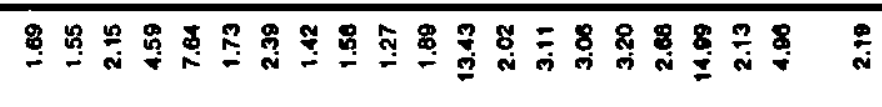 \\
\hline 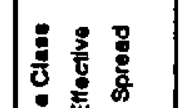 & 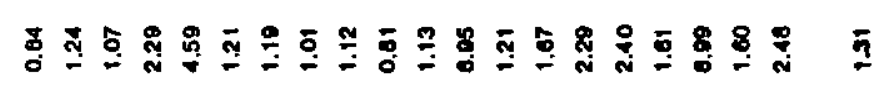 \\
\hline $\mid$ & 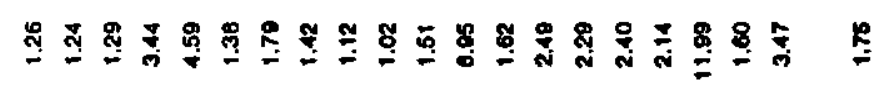 \\
\hline 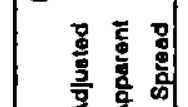 & 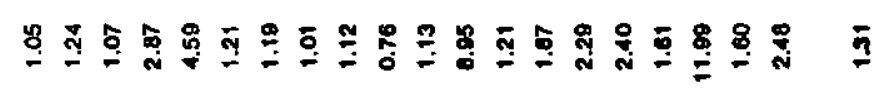 \\
\hline 害高 & 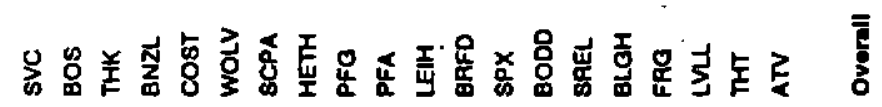 \\
\hline 总高高 & 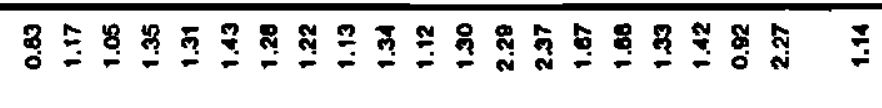 \\
\hline $\mid$ & 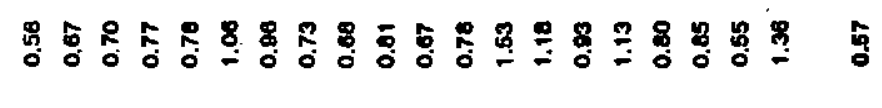 \\
\hline $\mid$ & 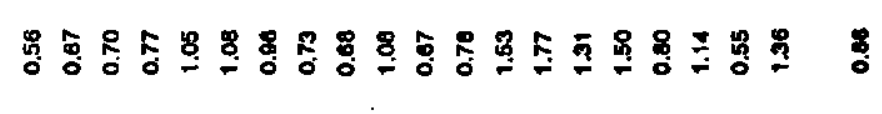 \\
\hline 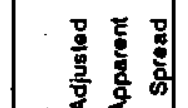 & 咅 \\
\hline 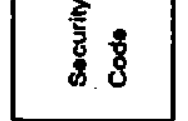 & 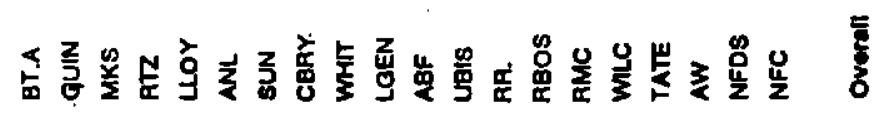 \\
\hline
\end{tabular}




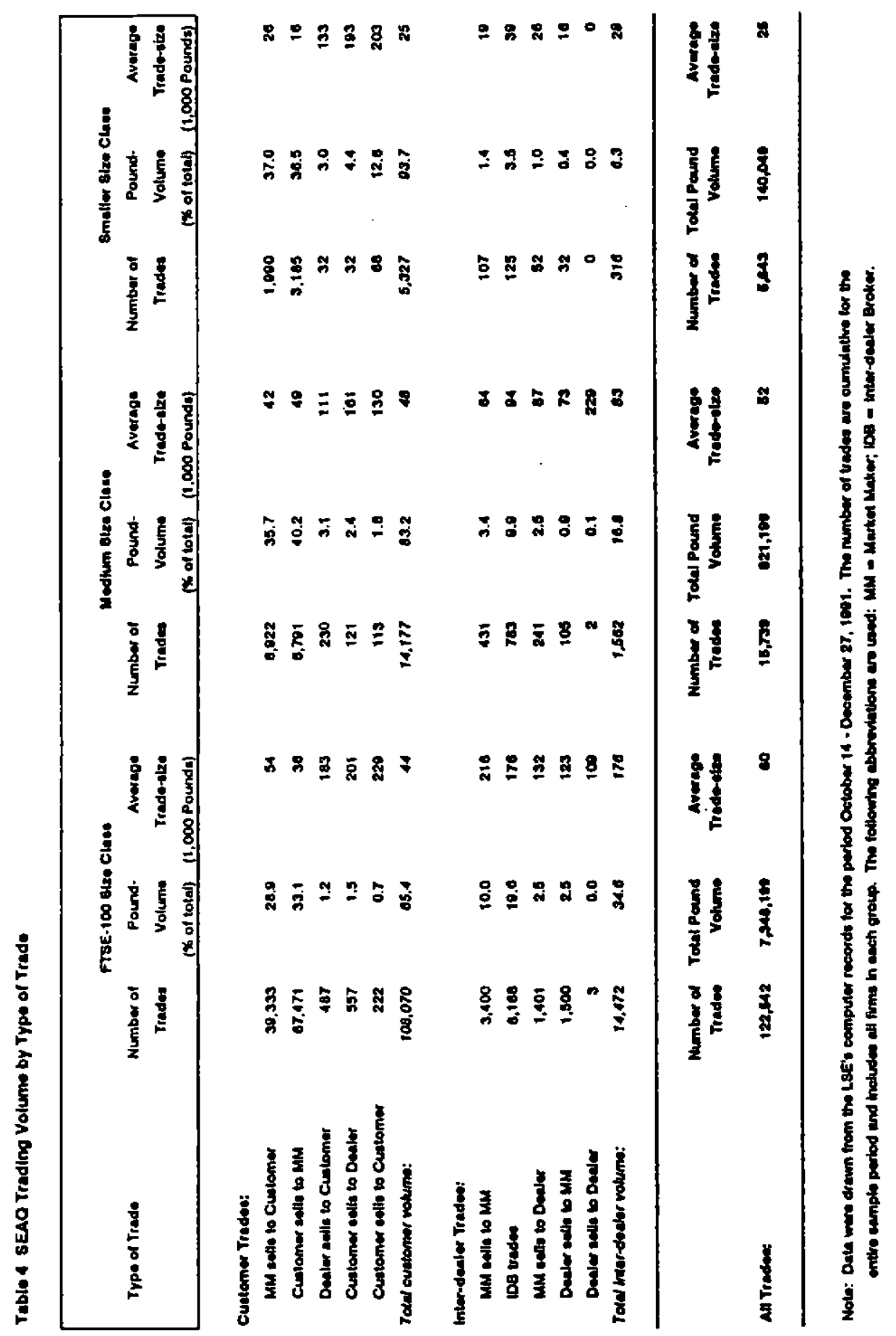




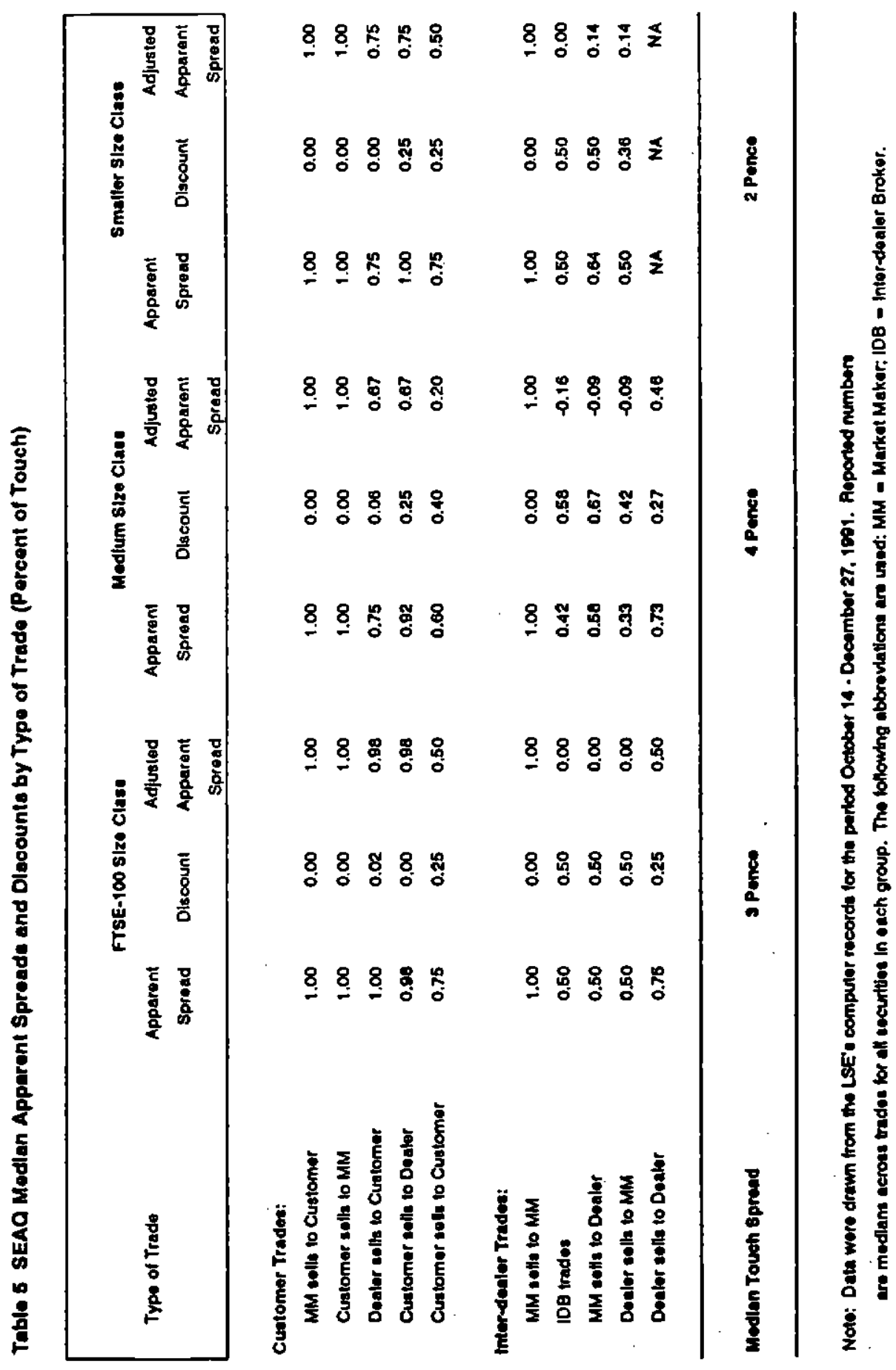




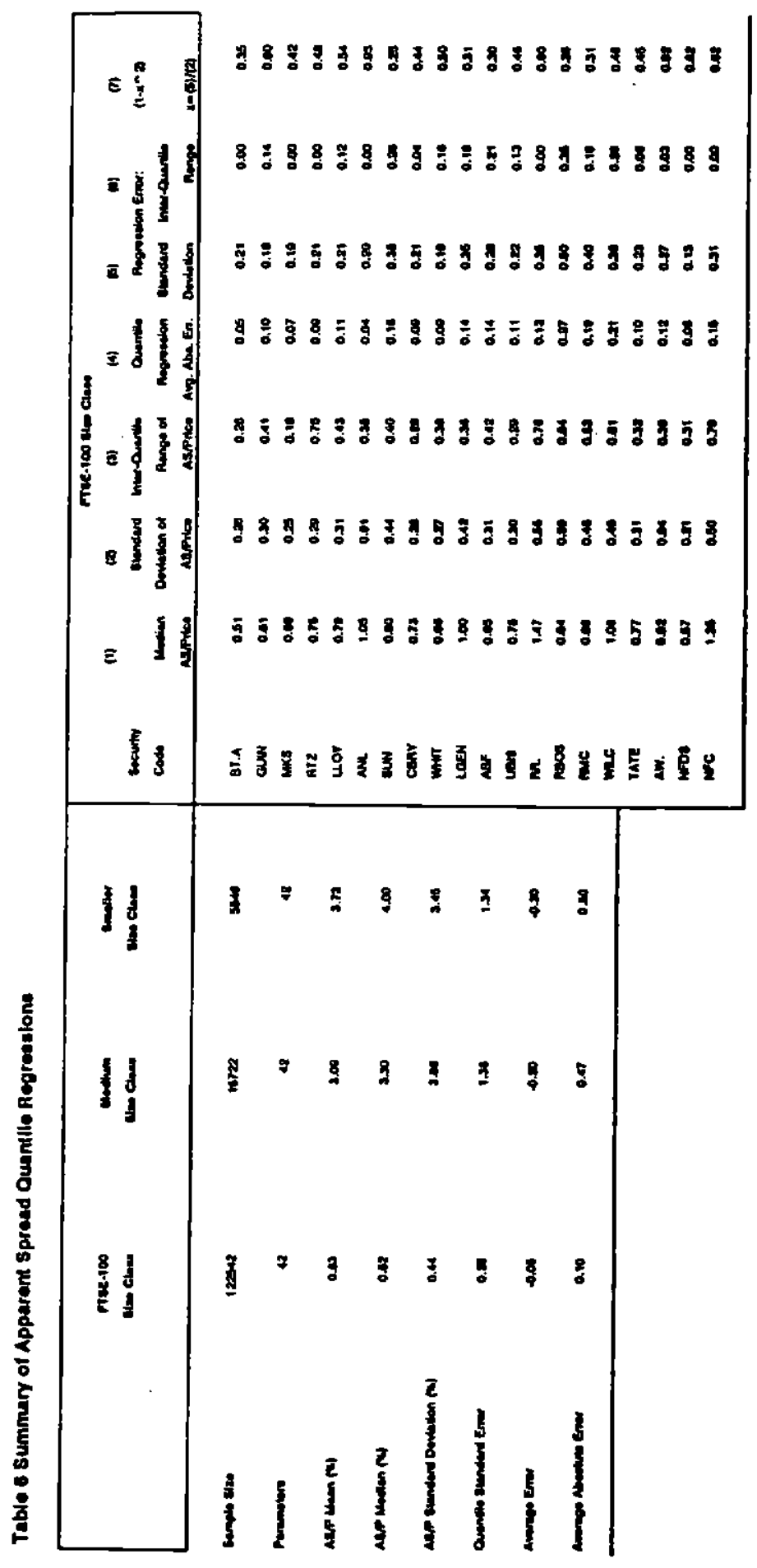




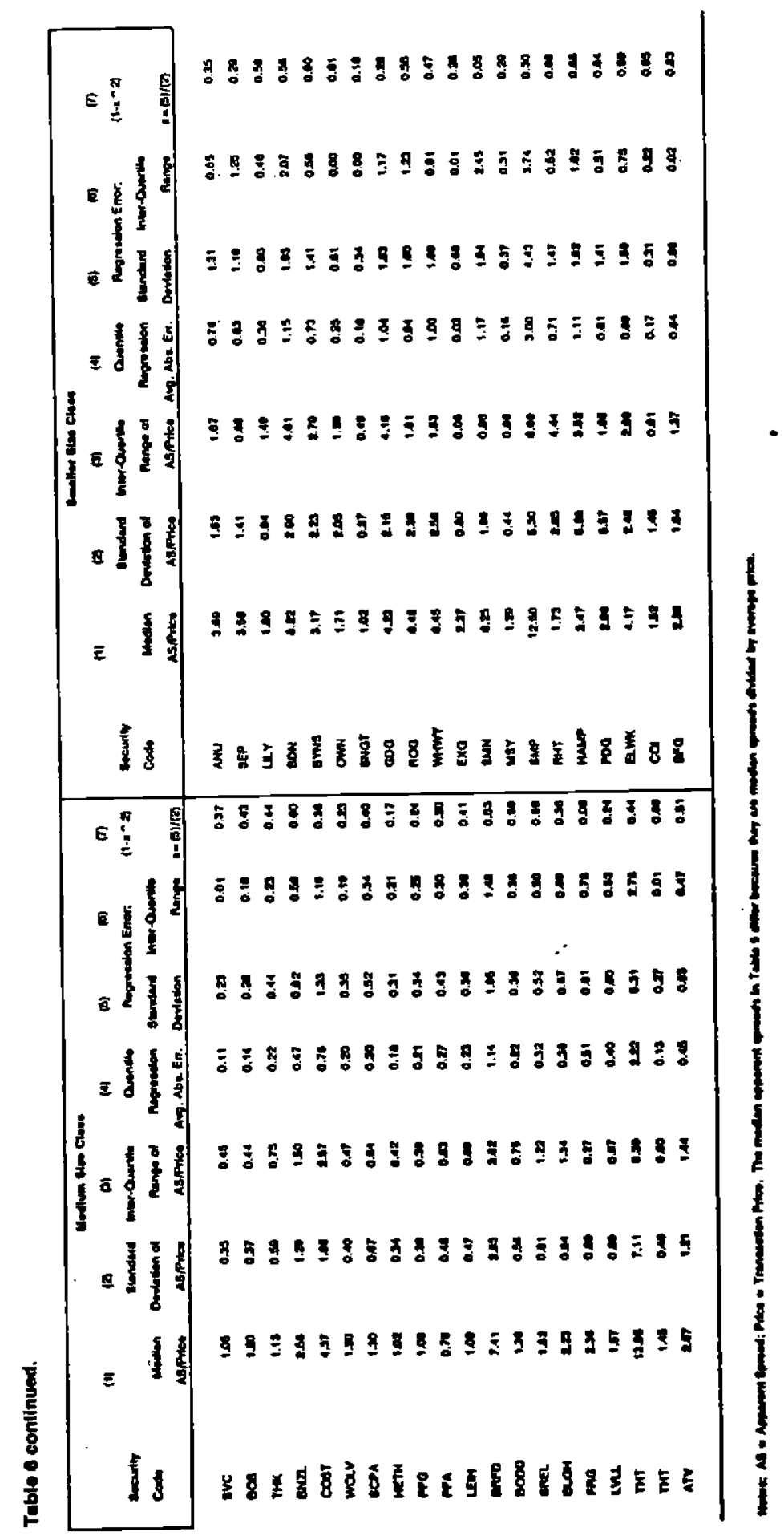




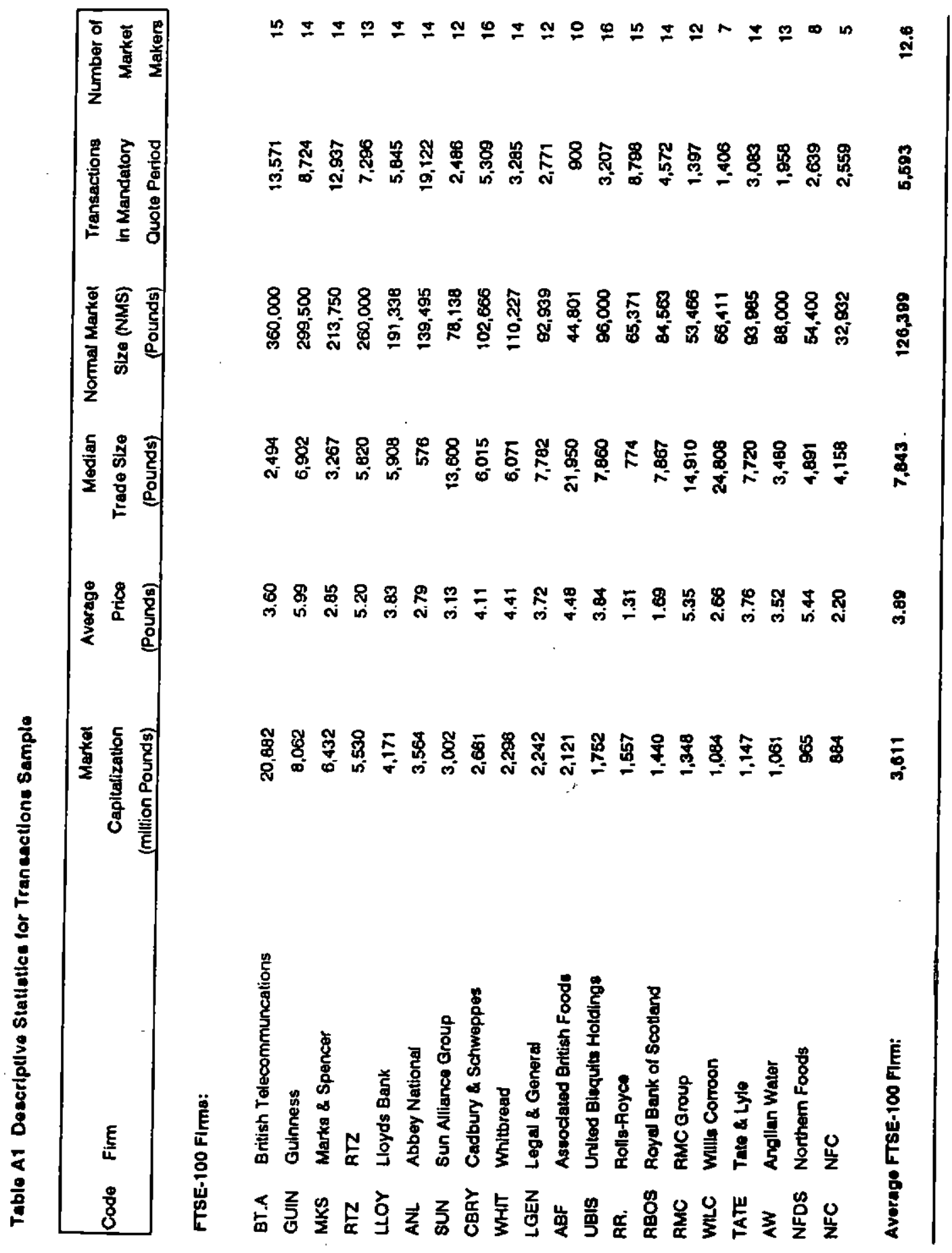




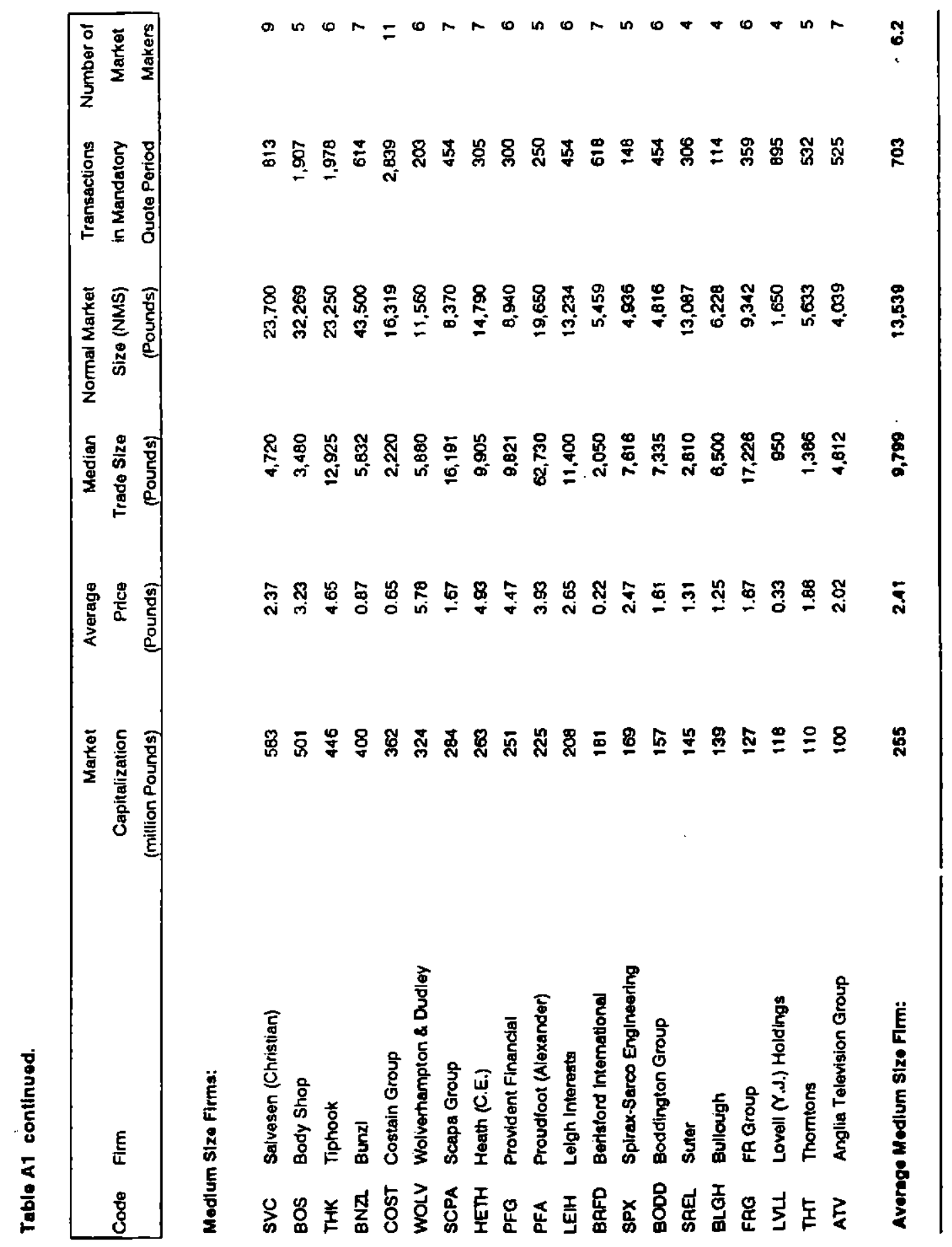




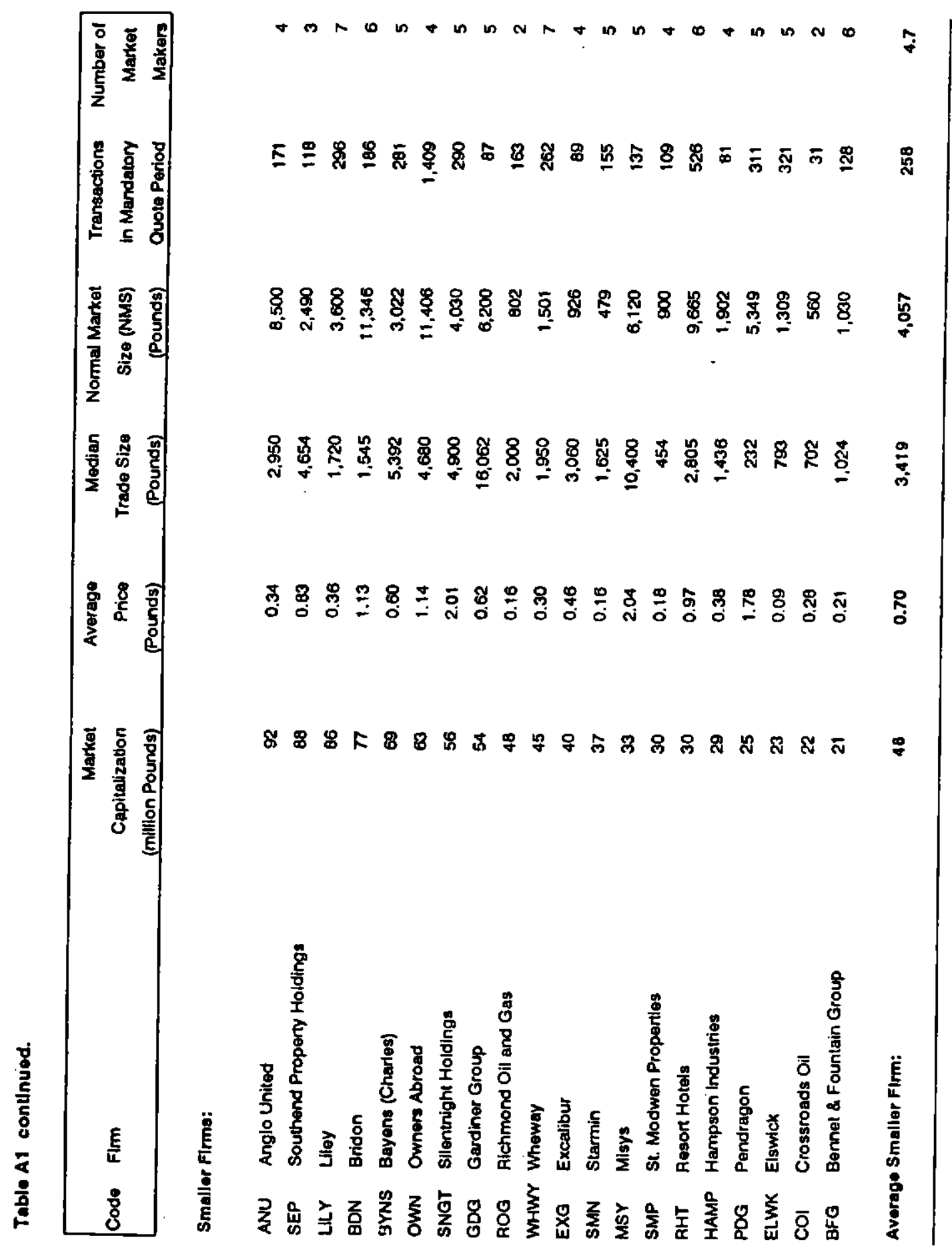




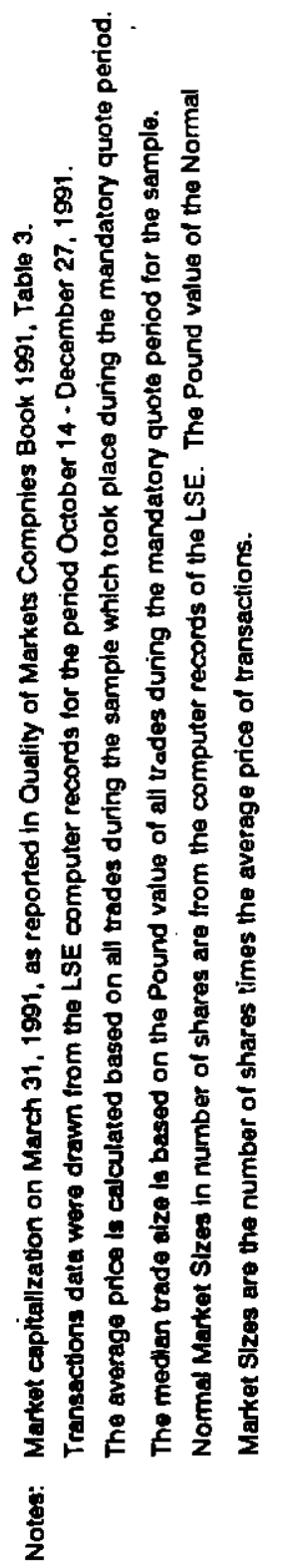


List of Figures

Figure 1a: Trade Types and Best Bid-Ask

Cadbury \& Schweppes - London

16 and 17 October 1991

(panels 1 and 2)

Figure 1b: Trade Sizes and Best Bid-Ask

Cadbury \& Schweppes - NASDAQ

16 and 17 October 1991

(panels 1 and 2)

Figure 1c: Trade Types and Best Bid-Ask

Cadbury \& Schweppes - London

October 16 and 17, 1991

(panels 1 and 2)

Figure 1d: Trade Sizes and Best Bid-Ask

Cadbury \& Schweppes - NASDAQ

October 16 and 17,1991

(panels 1 and 2)

Figure 2a: Conventional Spread Measures

Figure 2b: Adjusted Apparent Spread

Figure 3a: Apparent Spread by $\log ($ Trade Size) and Touch

Cadbury \& Schweppes - London 1991

Customer Trade Median AS/Touch

Figure 3b: Apparent Spread by Log(Trade Size)

Cadbury \& Schweppes - London 1991

Customer Trade AS/Touch Quartiles

Figure 3c: Apparent Spread by Log(Trade Size)

Cadbury \& Schweppes - NASDAQ 1991

Customer Trade AS/Touch Quartiles

Figure 3d: Apparent Spread by Log(Trade Size)

Cadbury \& Schweppes - London 1991

Dealer Trade Median AS/Touch

Figure 4a: Quoted Spread Quartiles by Number of Market Makers

1,887 SEAQ Ordinary Equities

Quartiles - Fourth Quarter 1991 
Figure 4b: Touch Spread Quartiles by Number of Market Makers

1,887 SEAQ Ordinary Equities

Quartiles - Fourth Quarter 1991

Figure 5: Apparent Spread by Log(Trade Size)

Medium Size Class Sample - Fourth Quarter 1991

Actual AS vs. Median Predicted AS

Figure 6a: Apparent Spread by Log(Trade Size) and Touch

FTSE-100 Size Class Sample - Fourth Quarter 1991

Customer Trade Median AS/Touch

Figure 6b: Apparent Spread by Log(Trade Size) and Touch

Medium Size Class Sample - Fourth Quarter 1991

Customer Trade Median AS/Touch

Figure 6c: Apparent Spread by Log(Trade Size) and Touch

Smaller Size Class Sample - Fourth Quarter 1991

Customer Trade Median AS/Touch

Figure 6d: Apparent Spread by Log(Trade Size)

FTSE-100 Size Class Sample - Fourth Quarter 1991

Dealer Trade Median AS/Touch 


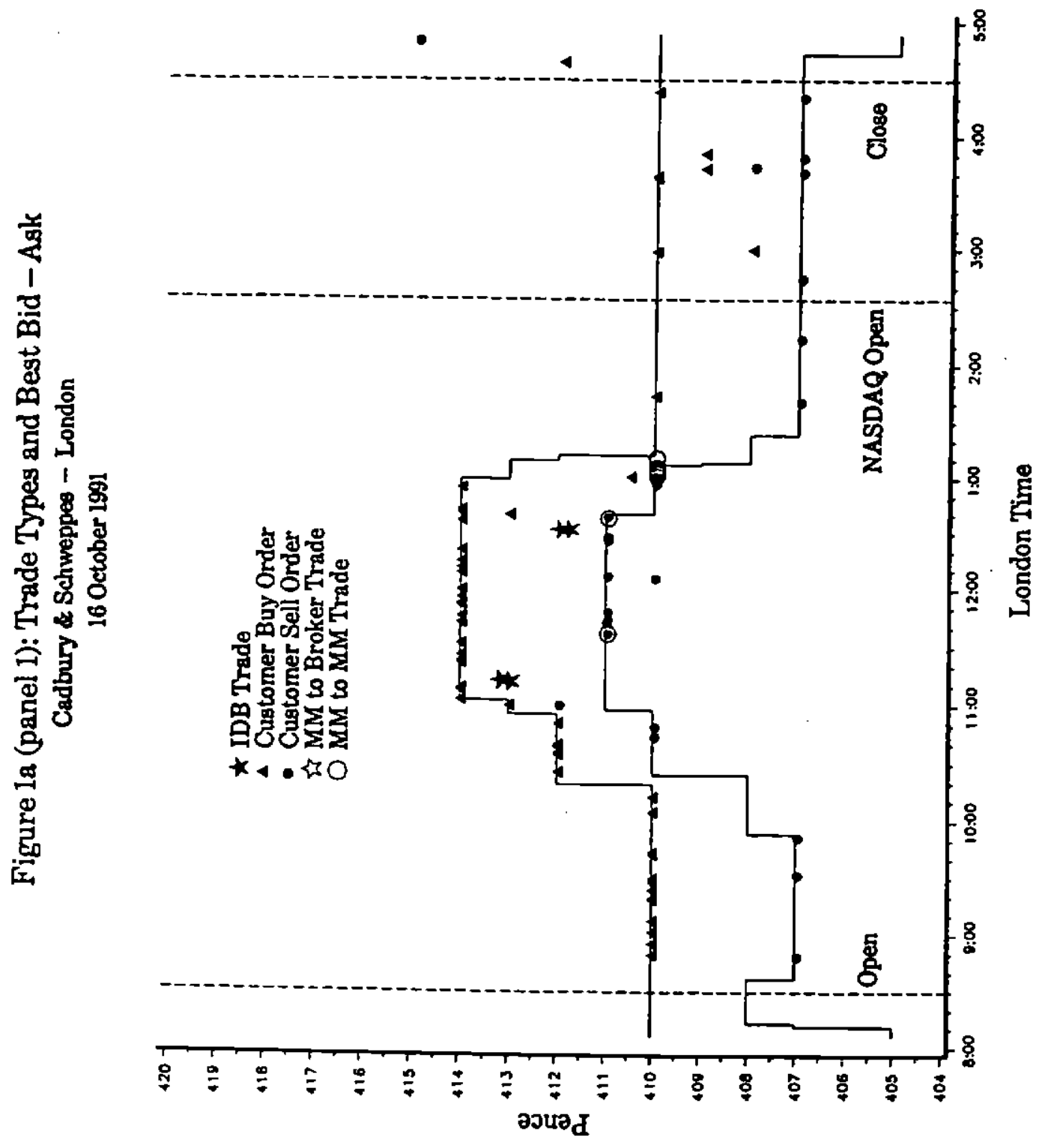




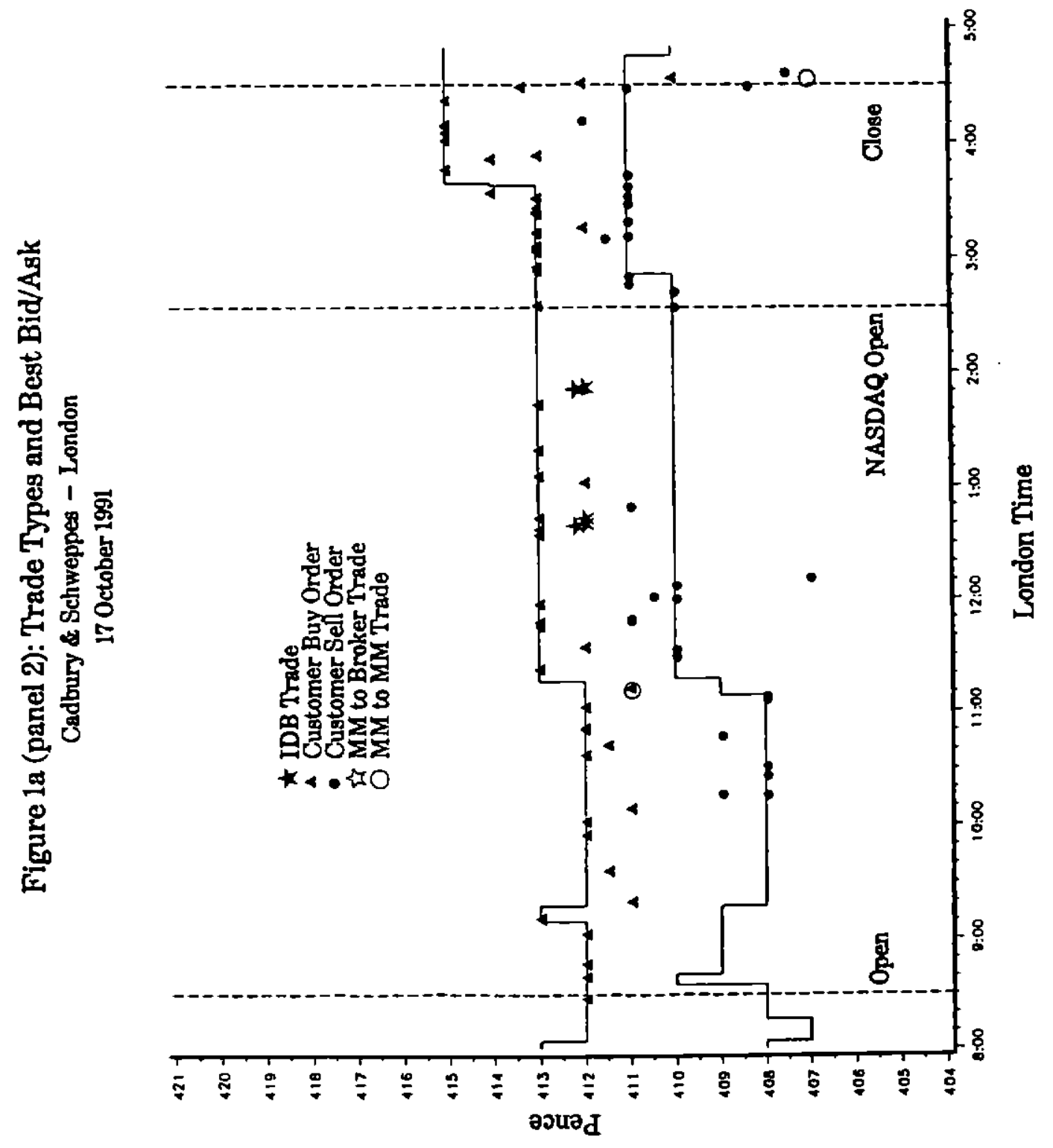




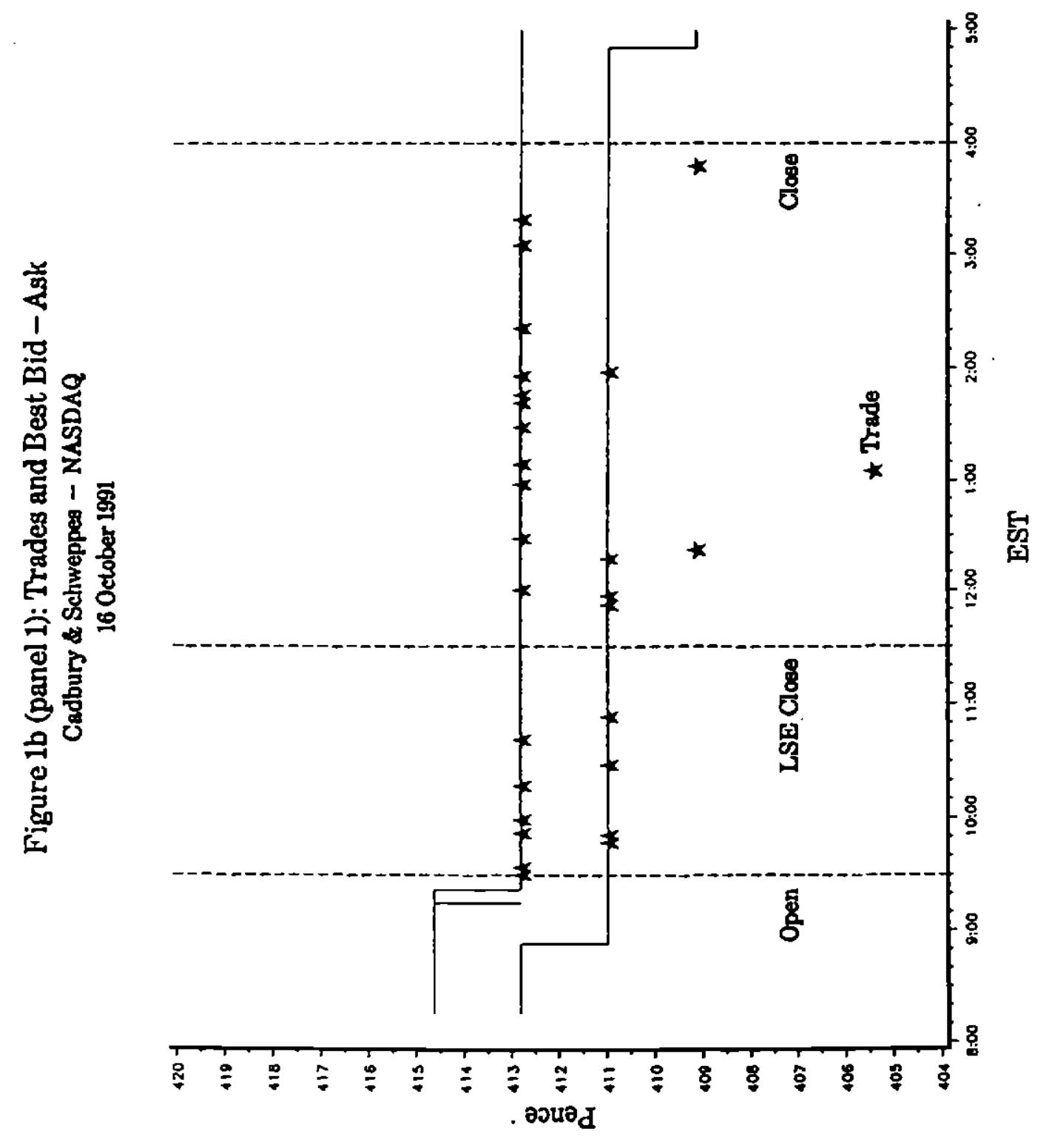




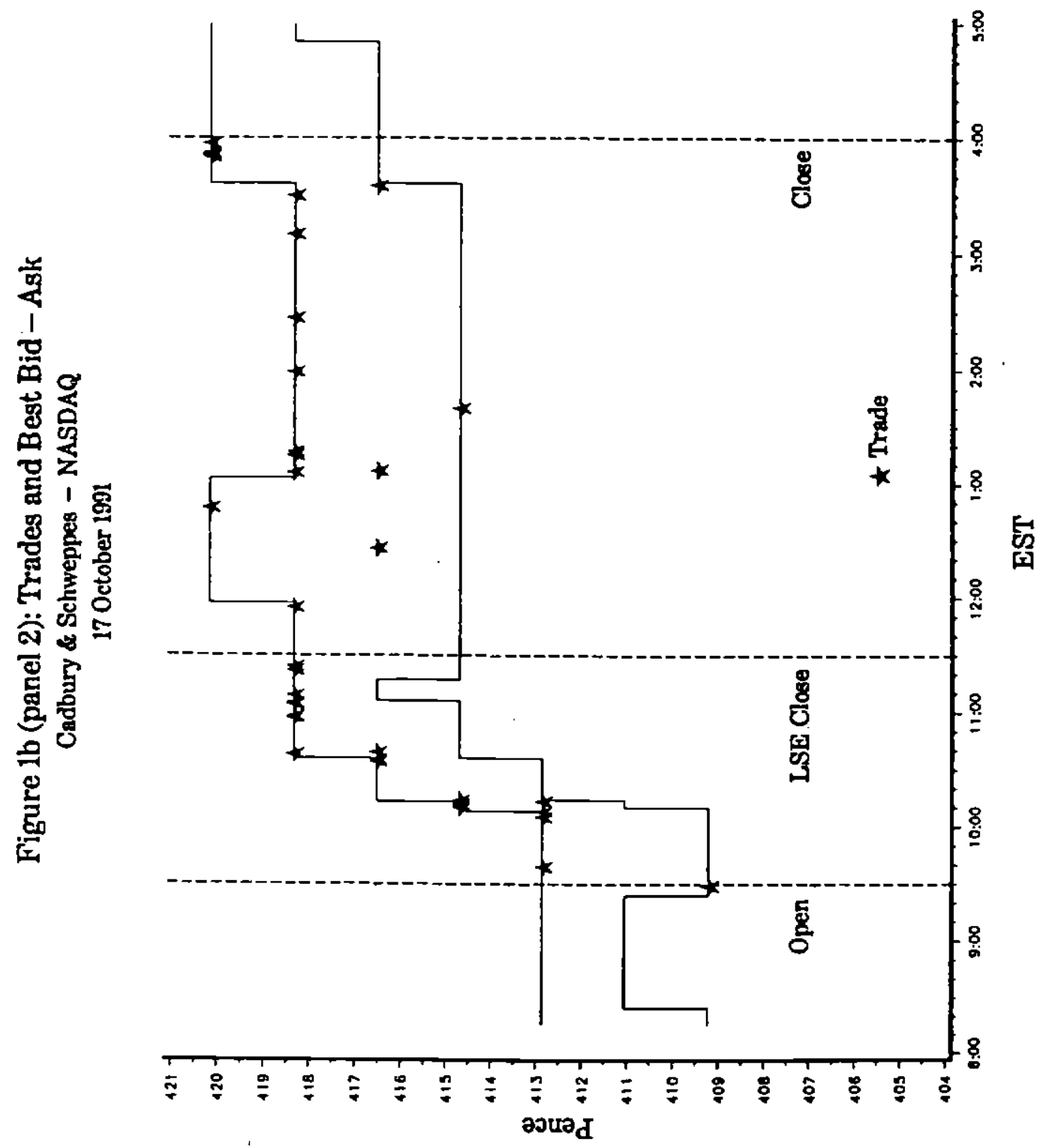




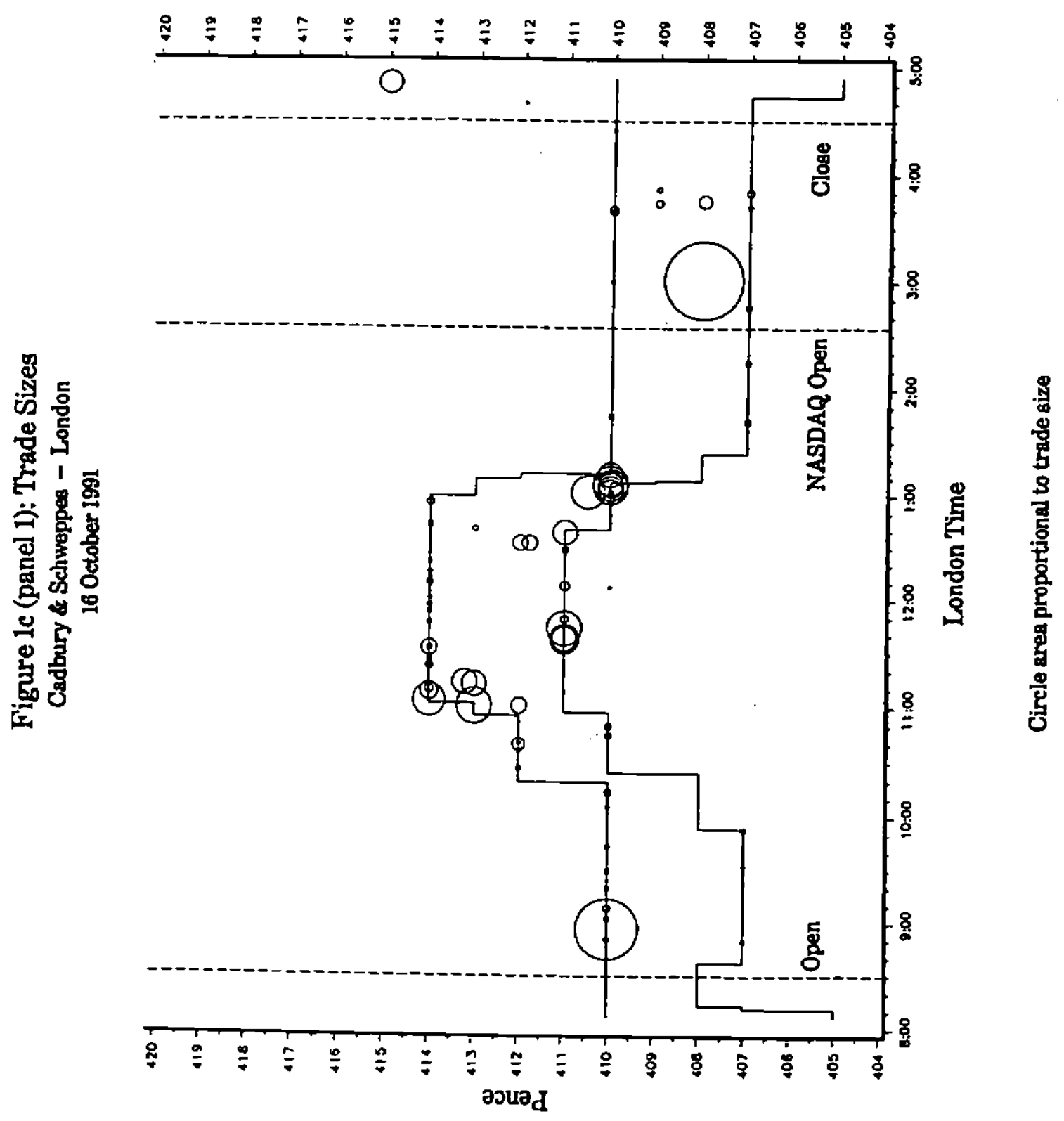




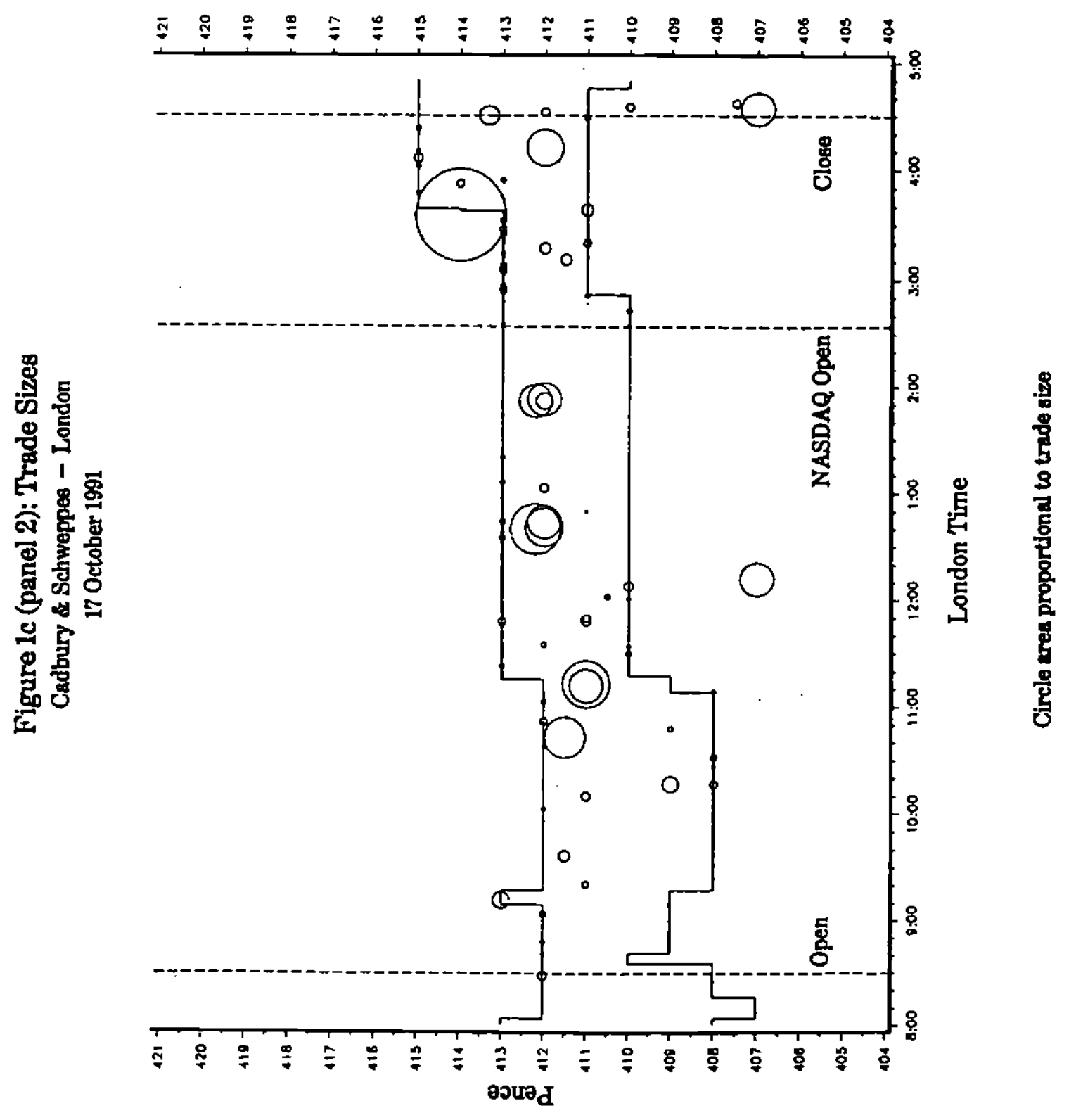




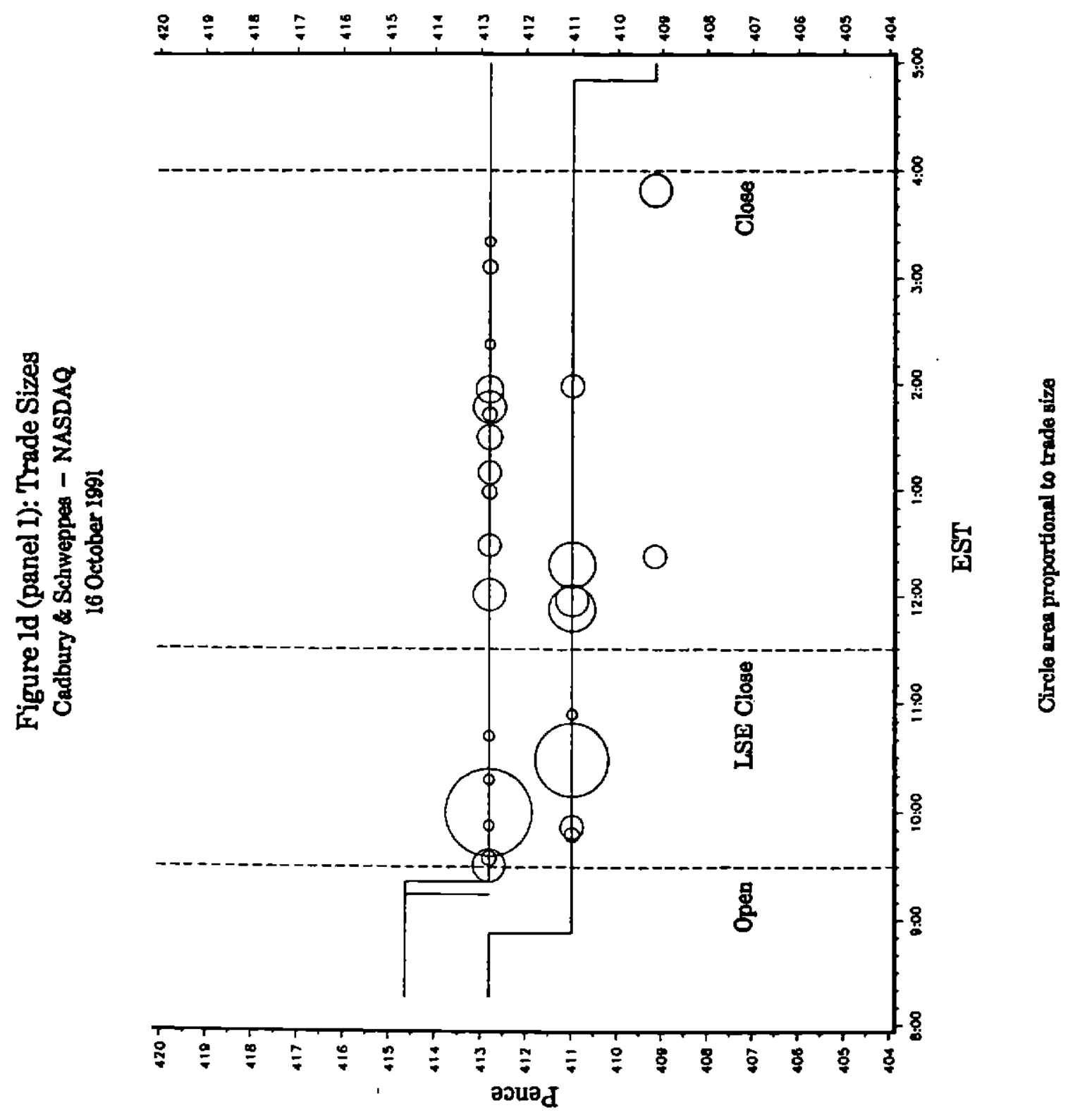




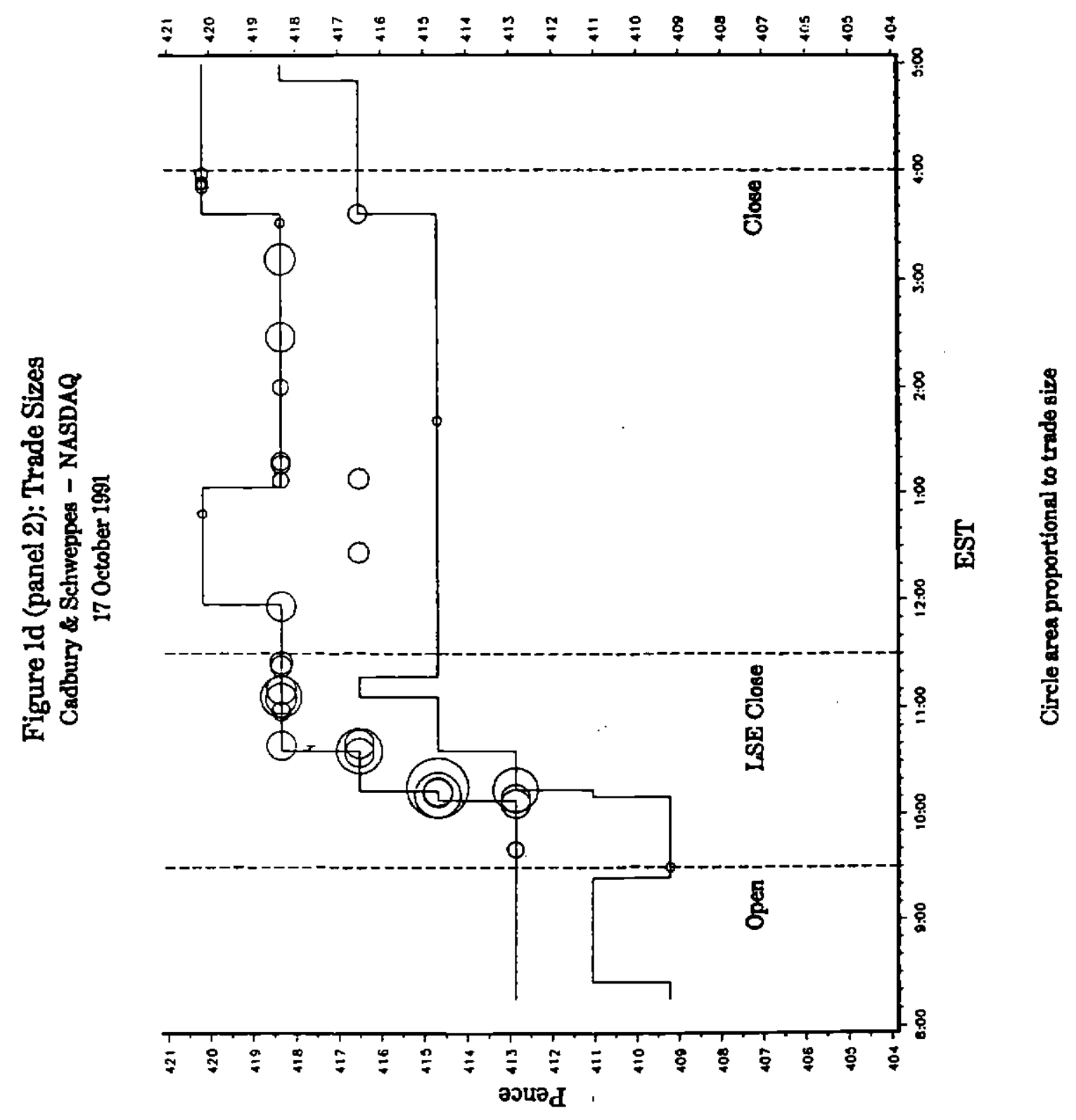


Figure 2a

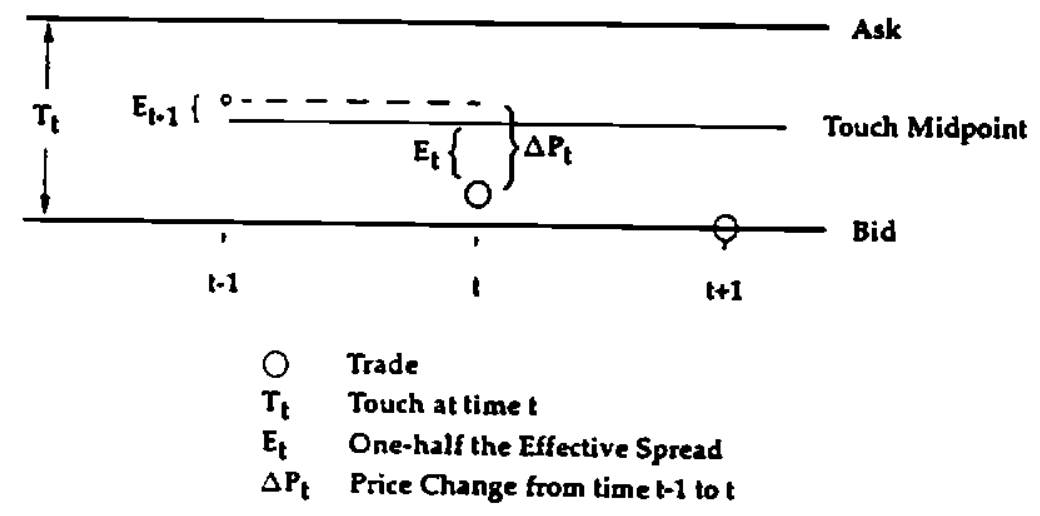

Figure 2b

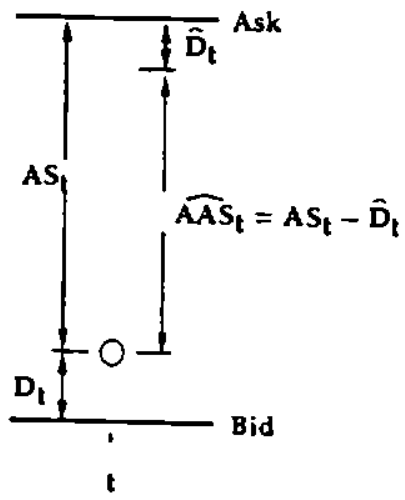

O Trade

AS Apparent Spread

$\widehat{A A S}$ Adjusted Apparent Spread

$D_{t}$ Trade Discount at time t

$\hat{D}_{1} \quad$ Estimated Trade Discount on Reverse Transaction 


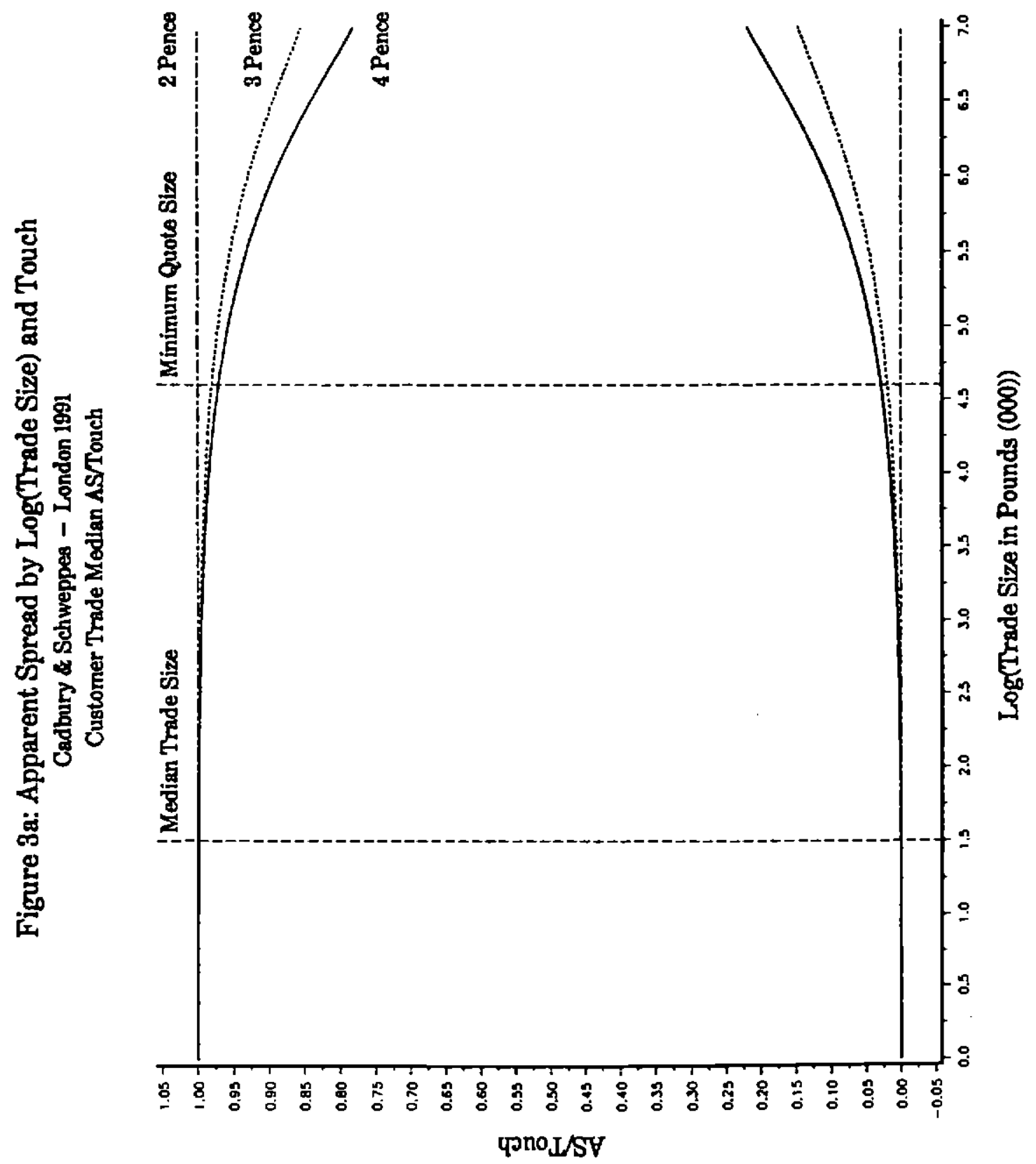




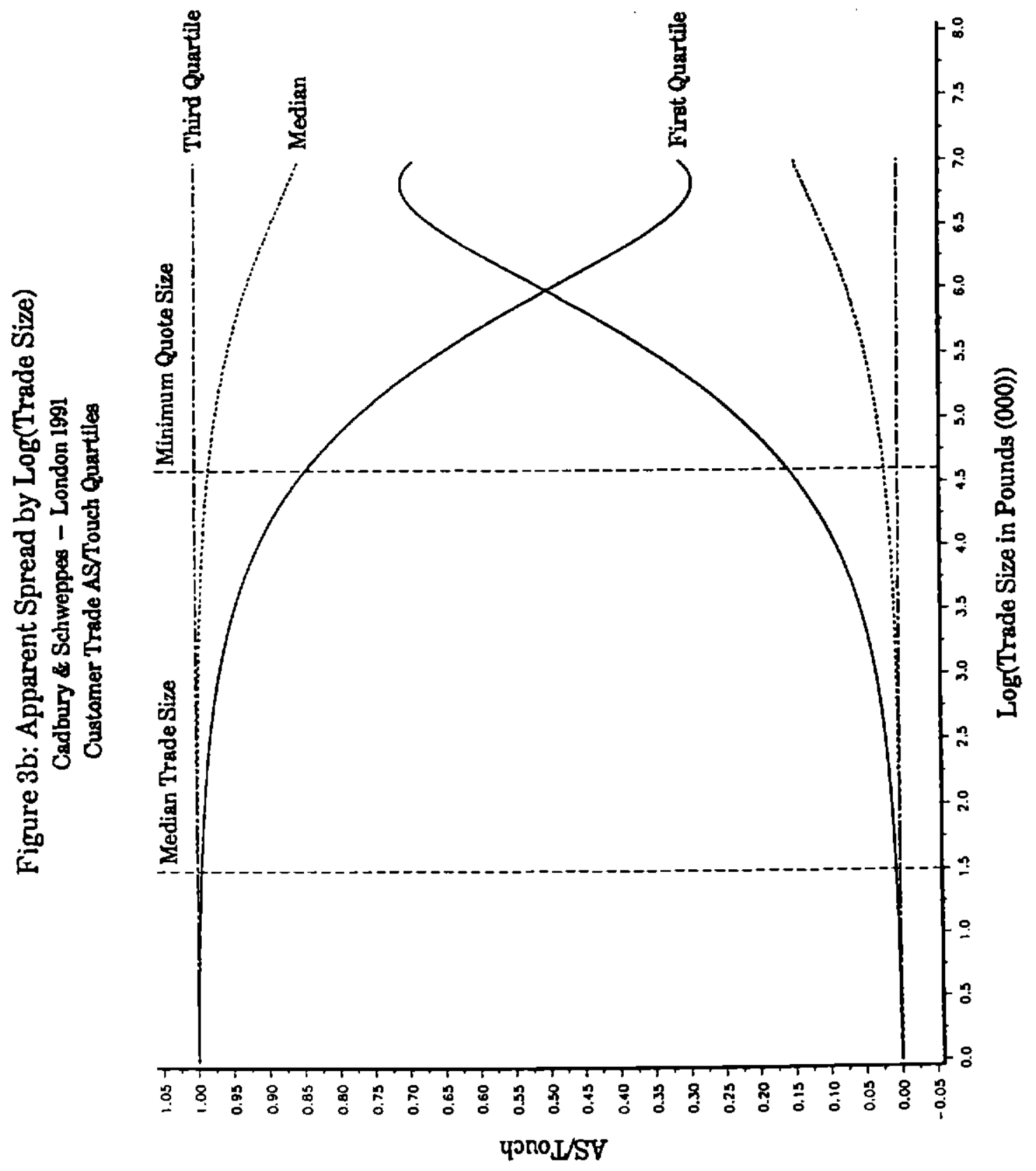




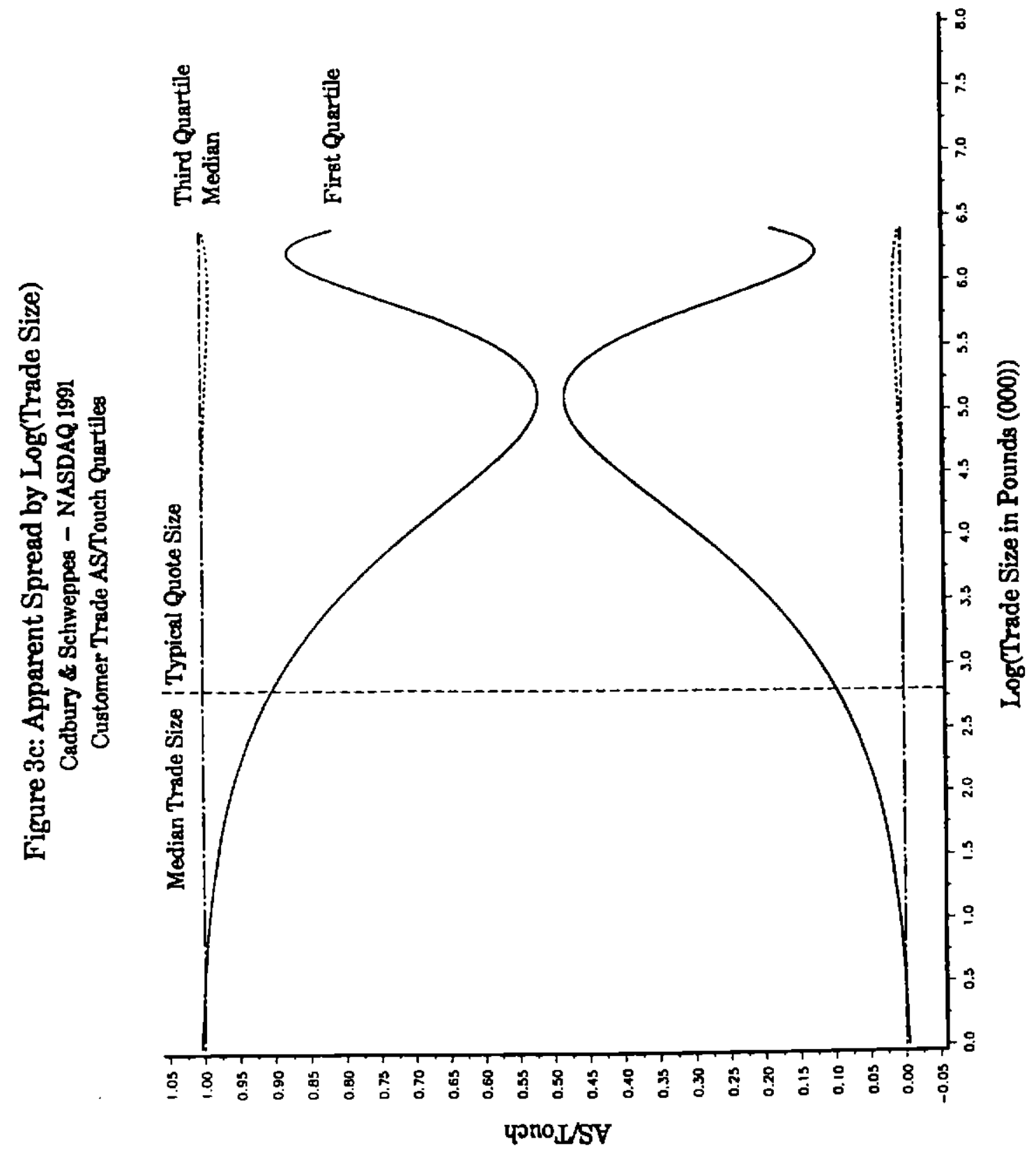




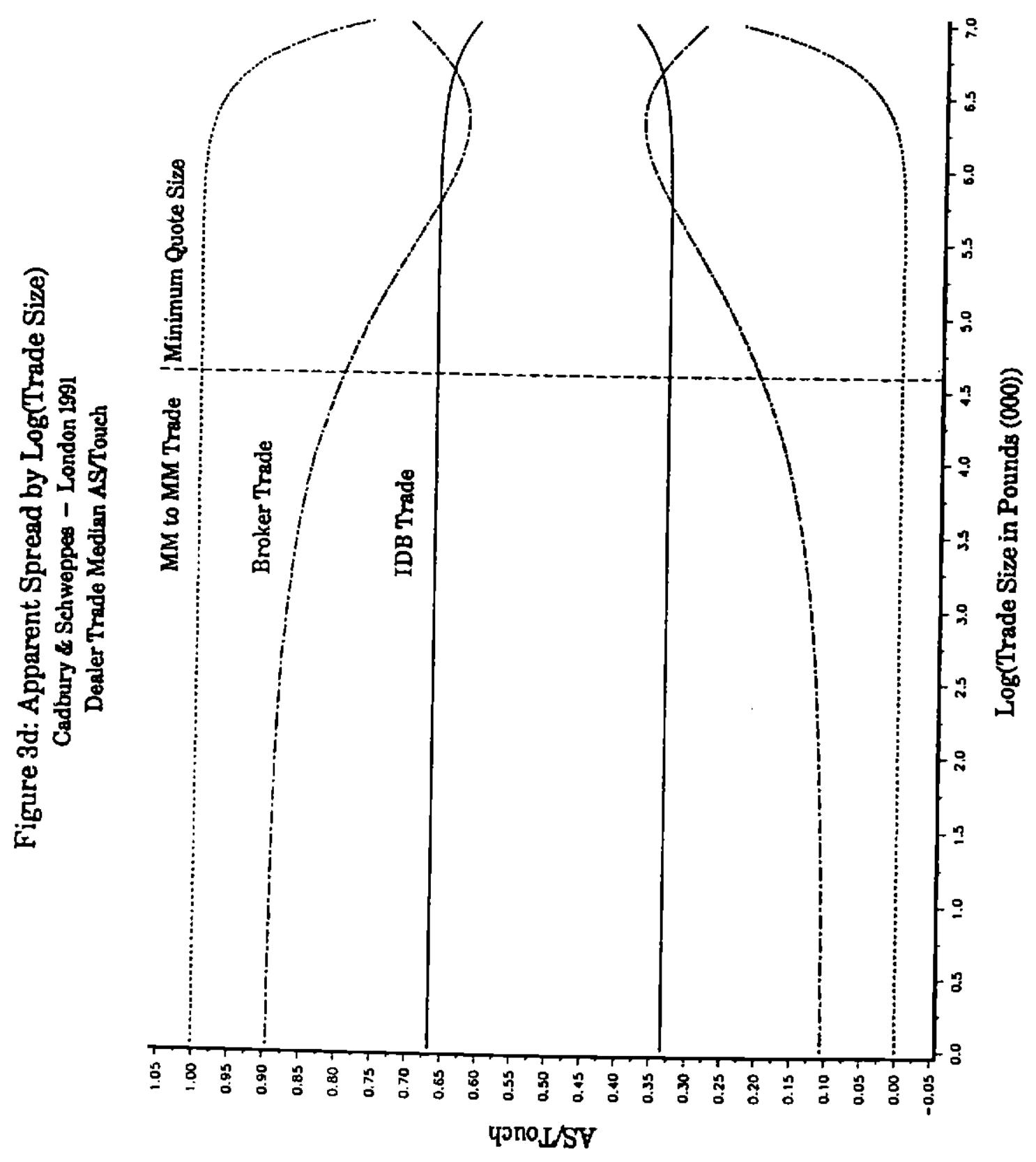




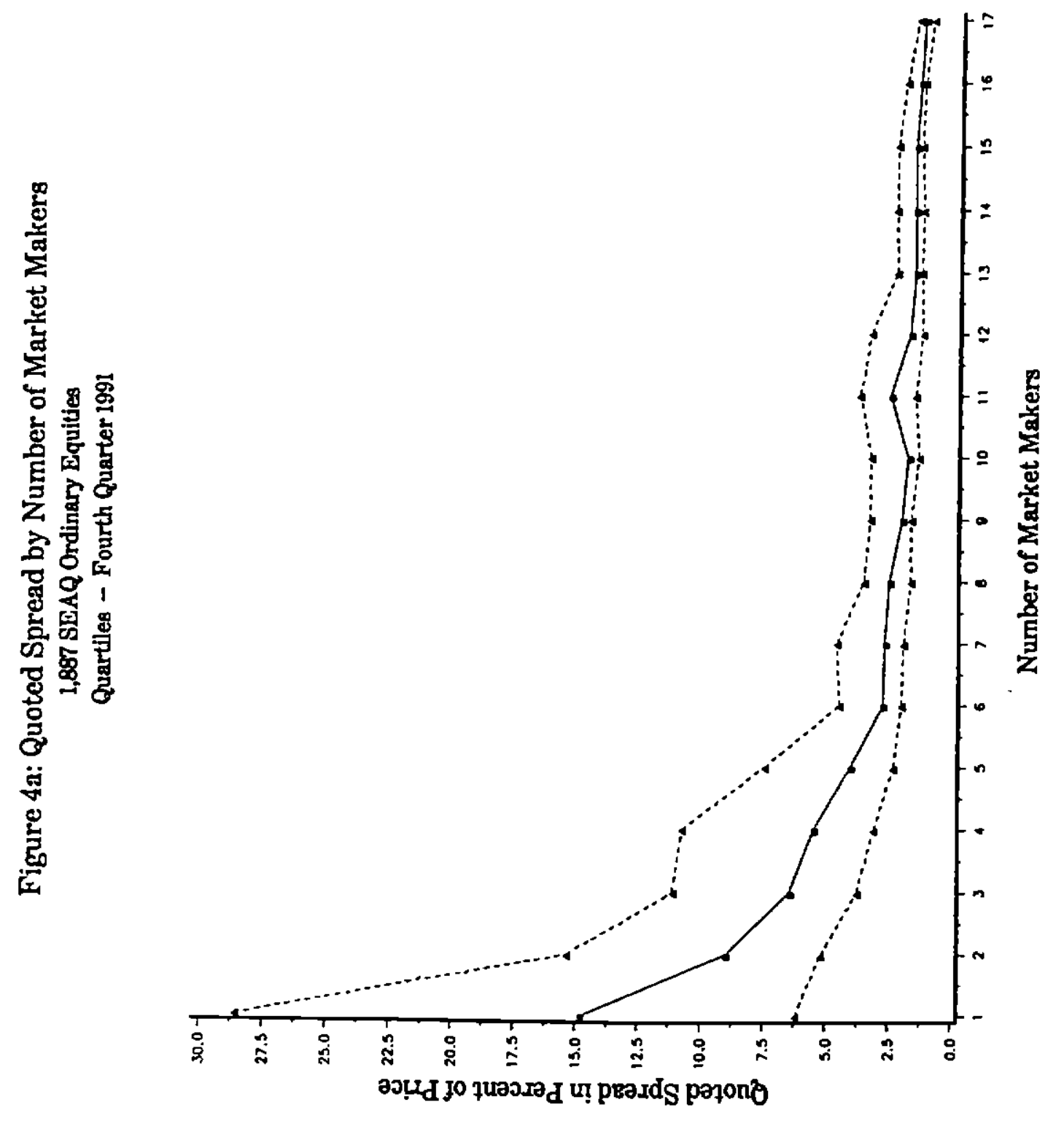




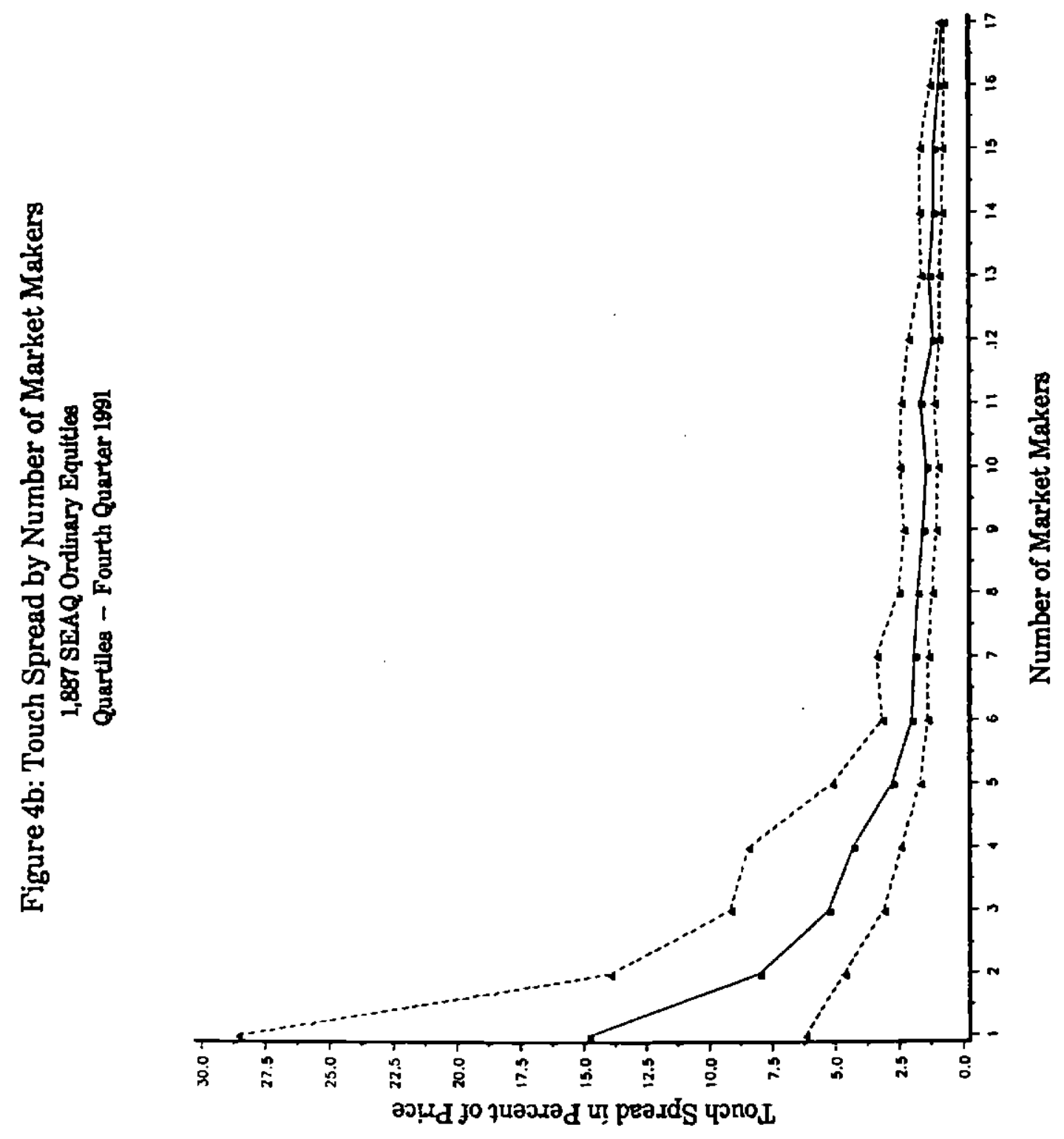




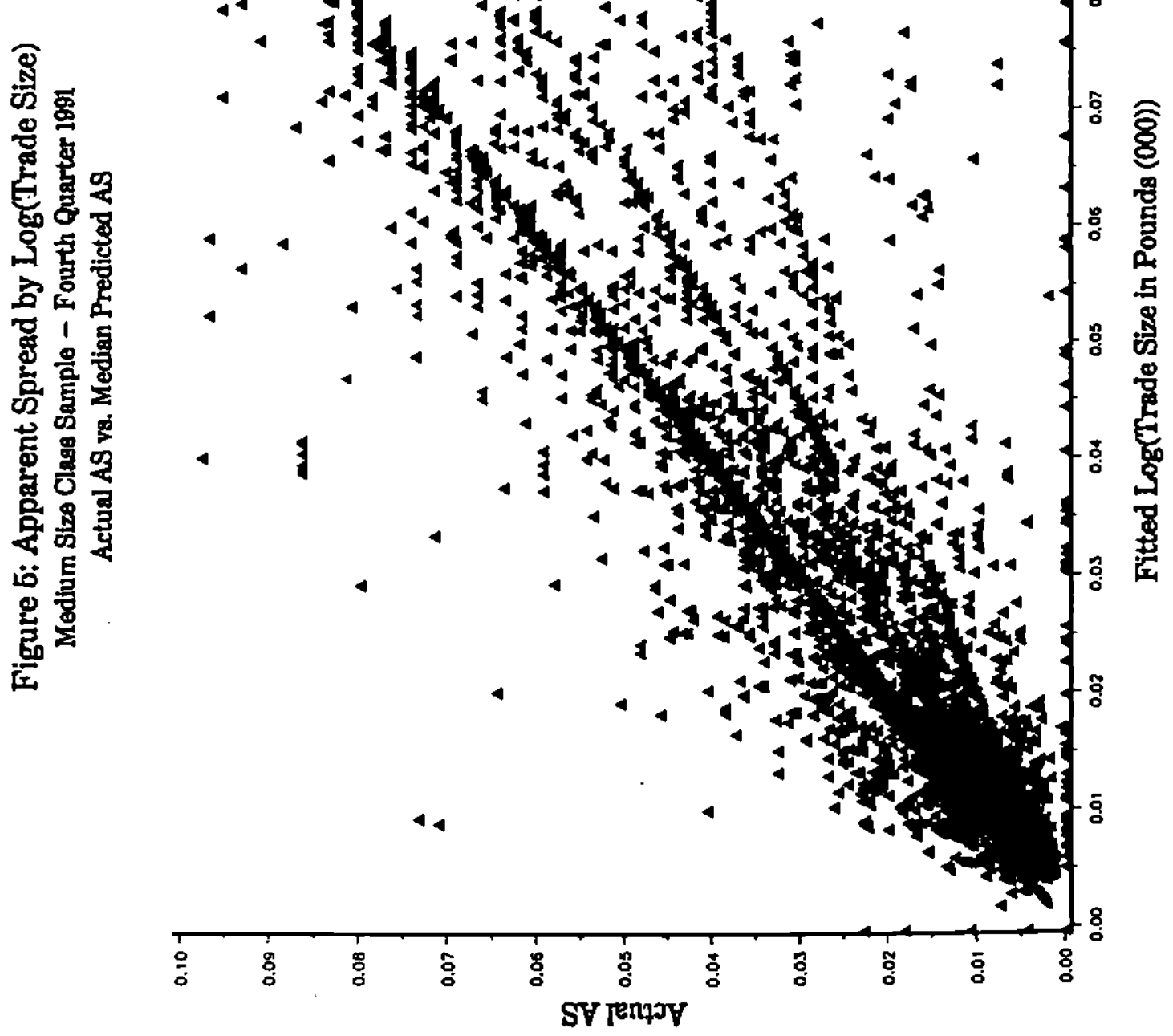




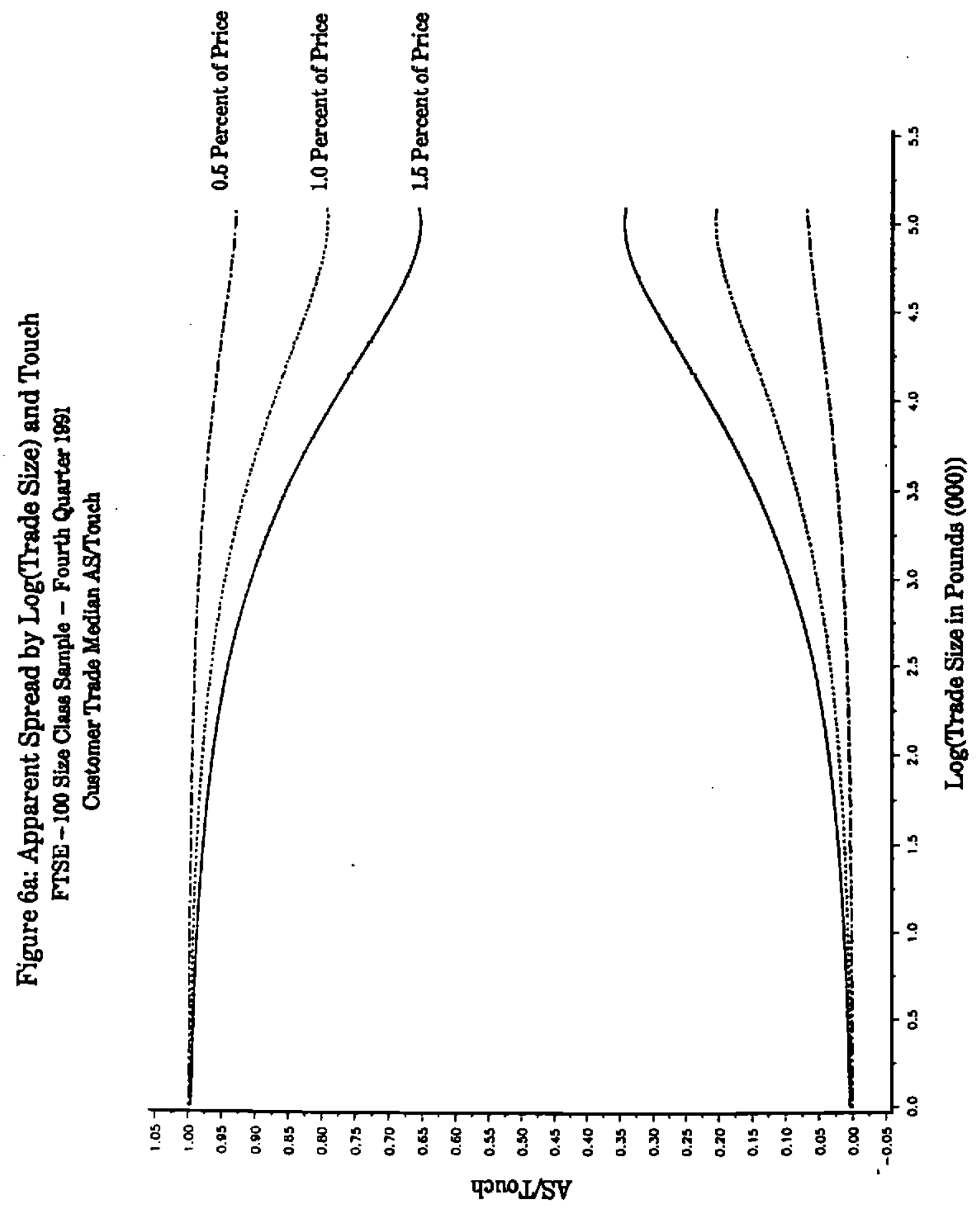




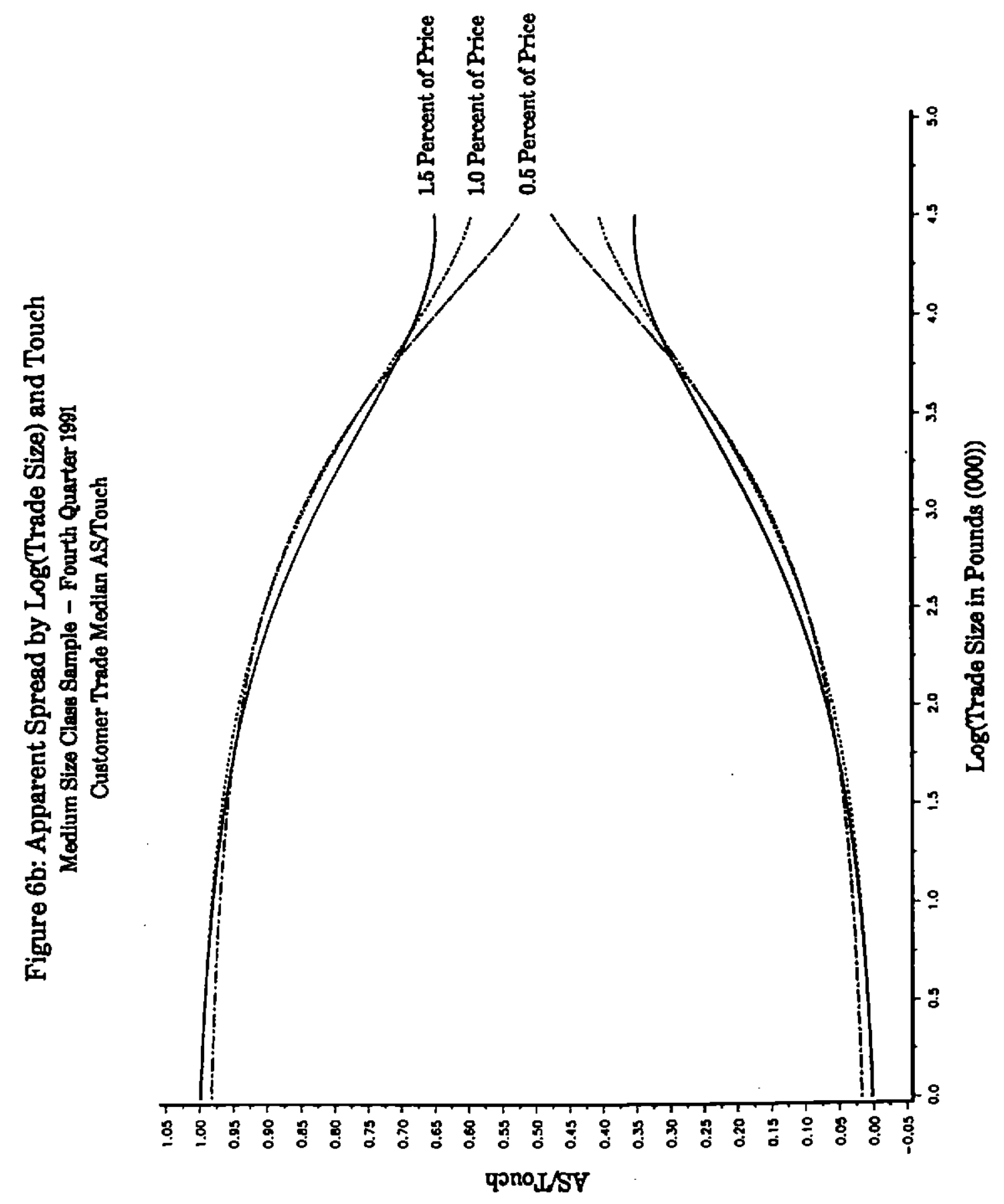




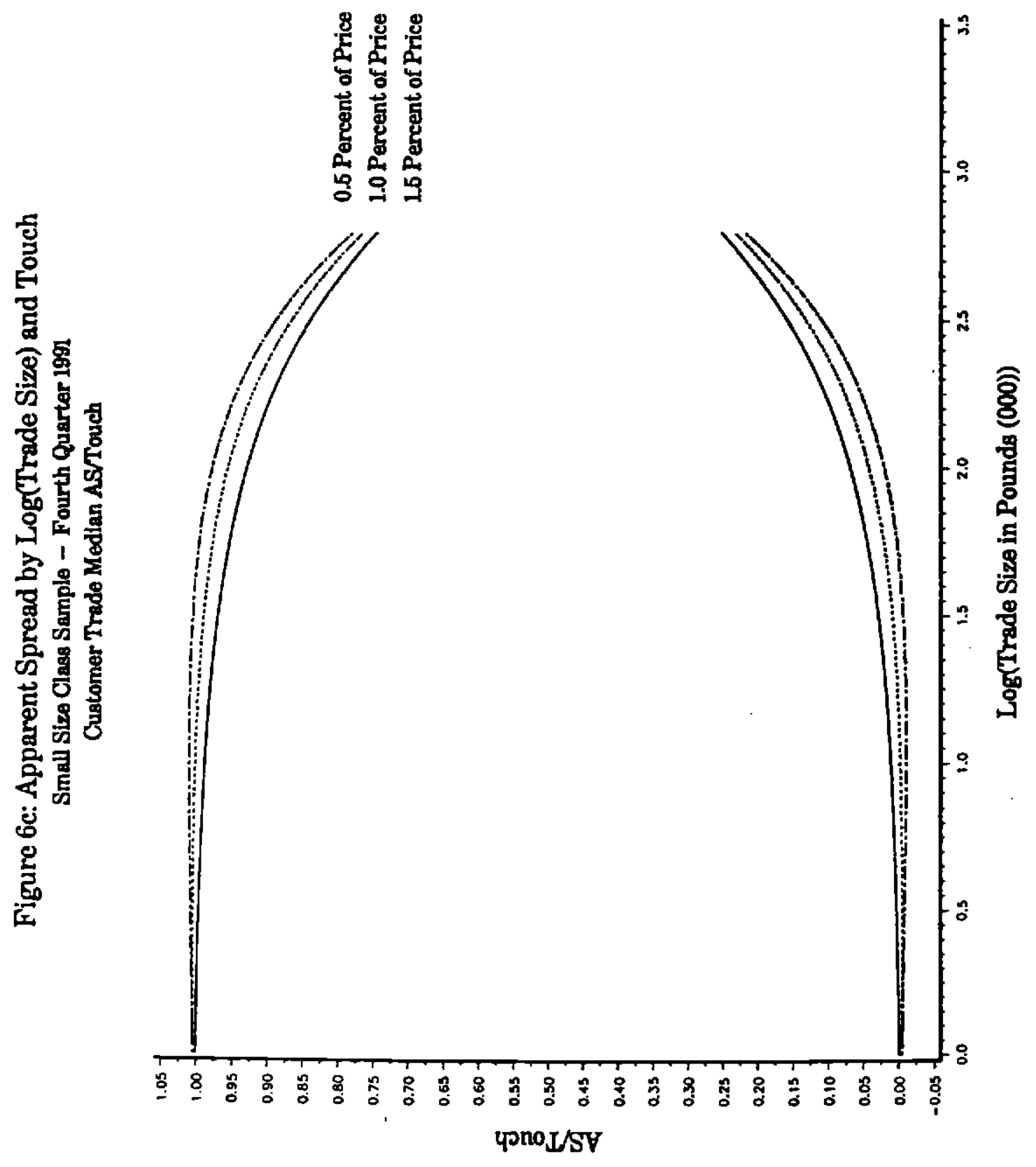




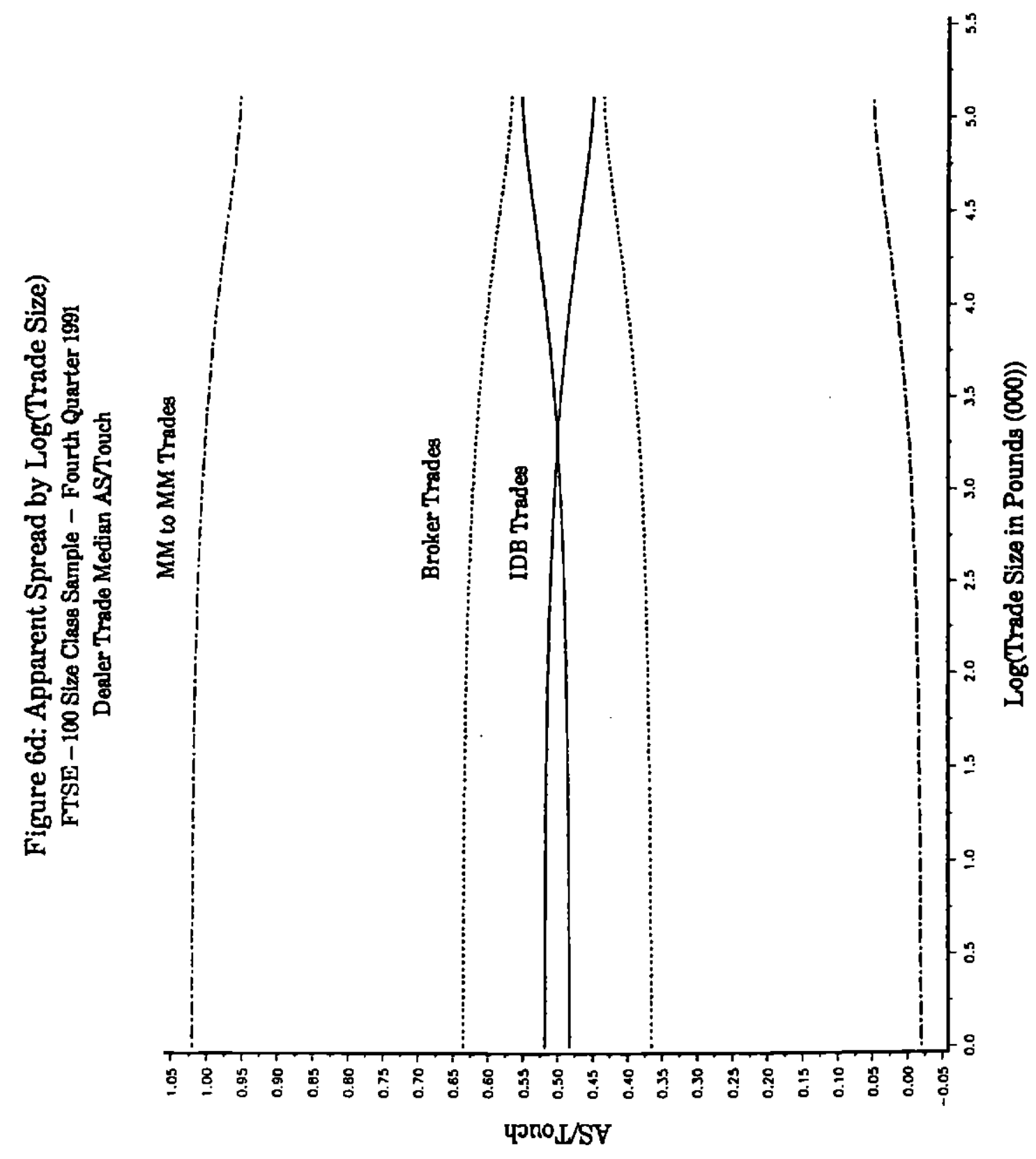

